\title{
20. Money Income Distribution and Redistribution in Australia, Sweden and the United States 1984'
}

\author{
Ian Castles
}

This paper compares the distribution of household money incomes in Australia, Sweden and the United States, and examines the association between the private incomes and cash benefits received and the direct taxes paid by various household groups within each of the three countries.

Over the years, many attempts have been made to compare the distribution of the money incomes of families in Australia with the corresponding distribution in other countries. In a review of the literature published in 1978 in the Surveys of Australian Economics series for the Academy of Social Sciences in Australia, Richardson commented:

The hazards of international comparisons of income distribution are severe. Briefly these hinge on the non-comparability of definitions of family and income; the possibility of substantial sampling error; and the inability of any existing measure of private income to reflect the value of government-provided goods and services. It is plain that even the most careful analysis should be taken as suggestive rather than conclusive. Despite the difficulties and the very tentative nature of the conclusions, a number of studies attempt this comparison of family incomes. ${ }^{2}$

After summarising these studies, she concluded:

Despite the overwhelming consistency of the evidence suggesting Australia has a very equal distribution of income, reservations remain. ${ }^{3}$

During the subsequent decade, much new information on the distribution of incomes in Australia has become available. The Australian Bureau of Statistics (ABS) undertook surveys of income in 1978-79 and 1981-82, and a Household Expenditure Survey in $1984 .^{4}$

\footnotetext{
1 Presented to the 1987 ANZAAS Congress in Townsville on 24 August 1987. Published by the Australian Bureau of Statistics as a monograph. Except where otherwise indicated, all figures and tables in this chapter are Castles' own.

2 Richardson, S, 'Income Redistribution, Poverty and Redistributive Policies' in Gruen, FH (ed.) Surveys of Australian Economics. Volume 2: 26.

3 Ibid: 27.

4 Another income survey was conducted in 1985-86, but results are not yet available.
} 
The output from these surveys has eased some of the difficulties of comparisons between Australia and other countries in this area. The problem of noncomparability of definitions of 'family' between countries has been diminished by the release of Australian data on various bases - income units, families and households; there is some evidence that the degree of non-comparability of estimates on a current income basis with those on an annual income basis may have been overstated; ${ }^{5}$ and steps have been taken towards adjusting private income measures to reflect the value of government-provided goods and services. ${ }^{6}$

Despite these advances, it remains the case that international comparisons of the distribution of household incomes are subject to problems of non-comparability of a kind that have long since been addressed - and largely overcome - in other statistical areas by the adoption of international conventions (e.g. the United Nations System of National Accounts, International Monetary Fund conventions for balance of payments statistics, International Labour Office conventions for statistics of employment, etc).

The present paper presents a comprehensive comparison of the distribution and redistribution of money incomes in Australia and Sweden on an income unit basis (that is, for units consisting of a couple or single person and their children if any), and some more limited comparisons between Australia and the United States on a household basis (that is, for units consisting of a group of people living together). For both studies, the method adopted was to match the published results from the 1984 surveys of money income in the overseas countries with corresponding data from the ABS Household Expenditure Survey 1984. The objective is to show how household survey data can be used to compare the structure of income distribution and redistribution between countries.

The detailed bilateral comparisons are contained in attachments to the paper. Although the Australia/Sweden and Australia/United States comparisons are on quite different bases, the data strongly suggest that the distribution of household incomes in Australia is less equal than in Sweden and more equal than in the United States. They also suggest that, although the purchasing power of money incomes in Australia is lower on average than in the United States and higher

\footnotetext{
5 As Table A in Attachment A shows, the distribution of household incomes on a current income basis from the 1984 Household Expenditure Survey was very similar to the corresponding distribution on an annual income basis from the 1981-82 Income and Housing Survey.

6 Estimates of the value of government-provided goods and services for Australia are given in Australian Bureau of Statistics Household Expenditure Survey Australia 1984: The Effects of Government Benefits and Taxes on Household Income (ABS Cat. No. 5237, April 1987). Estimates for Australia, New Zealand and the United Kingdom are compared in Castles, I, 'The Effects of Government Benefits and Taxes on Household Income: Estimates for Australia and Other Countries' in Saunders, P (ed.) 'Redistribution and the Welfare State: Estimating the Income Effects of Government Benefits and Taxes on Household Income'. Social Welfare Research Centre Reports and Proceedings, No. 67, August 1987.
} 
on average than in Sweden, the real value of the money incomes of low-income households in Australia is higher than in the United States and lower than in Sweden.

As this example shows, estimates of relative average real income levels for entire populations are of limited value in assessing the real incomes of specific groups within countries. Other illustrations of the limitations of global comparisons of real income levels will emerge from the discussion of the main findings of the Australia/United States studies in this paper.

\section{Money incomes of income units in Australia and Sweden 1984}

Statistics Sweden has conducted an 'Income Distribution Survey' annually since 1973. The income units used in the survey are described as family units, and are defined as follows:

Family units consist of cohabitants (married or unmarried) with or without children less than 18 years old, or single persons with or without children less than 18 years old. ${ }^{7}$

The ABS Household Expenditure Survey 1984 (HES) was, as the name implies, a survey of households (spending units) rather than of income units. For purposes of this section of the paper, the income data collected for individual members of households has been aggregated to produce data for 'income units', which are identical to 'family units' as defined in the Swedish income distribution surveys. ${ }^{8}$

The detailed tables in Attachment A present comparable information for income units (IUs) from the Australian HES and from the Swedish Income Distribution Survey 1984. The tables make use of concepts and classifications of income, income units, income unit quantiles, consumption units and real values. These are defined and explained in the next section.

7 Statistics Sweden, Statistical Series Be 21 SM 8601, Income Distribution Survey in 1984: Income Development of Families in 1975-1984: 16. The main results of the 1984 Income Distribution Survey are published in this publication and in Statistical Series Be 21 SM 8603, Income Distribution Survey in 1984: Income Distribution of Families in 1984.

8 'Income unit' as defined here is similar to, but not identical with, the definition used by the ABS in its income and expenditure surveys. For the latter definition see, for example, ABS Household Expenditure Survey 1984: the Effects of Government Benefits and Taxes on Household Income: 80. 


\section{Concepts and classifications}

Two concepts of income are used in the comparisons: 'private income' and 'disposable income'. 'Private income' includes all money receipts, other than government pensions and benefits, which are received regularly by any member of the income unit. 'Disposable income' represents private income plus direct benefits received less direct taxes paid. ${ }^{9}$ In some of the tables the net difference between private income and disposable income ('net cash transfers') is disaggregated into 'direct benefits received' and 'direct taxes paid'.

The comparisons are made for income units in three age groups: $18-19$ years; 20 64 years; and 65 years or more. Most of the tables relate to the '20-64 years' age group. In this age group, income units are classified into four broad categories:

1. married couple without children;

2. married couple with children;

3. single parents;

4. single persons;

and further subdivided according to the employment status of adult members and (for income units with children) the number of children. The ' 65 years or more' age group is subdivided into married couples and single persons.

The various populations of income units are divided into quantiles, so that the structure of incomes within those populations can be expressed by means of average values of private or disposable income within each quantile. The main quantiles used are 'deciles' - i.e. 10 per cent groupings of the estimated population when income units are ranked in ascending order according to each income unit's total private or disposable income. Many of the tables also show comparable average values for the 5 per cent and 2.5 per cent of the relevant income unit population with the highest incomes, and for the 5 per cent of the relevant population with the lowest incomes. The tables of distributions also show a 'co-efficient of concentration', which is an approximation of the Gini coefficient calculated from the average incomes for each decile.

In order to provide a rough adjustment of the 'income per income unit' data to take account of the differing needs of income units of varying size and

\footnotetext{
9 'Direct benefits' represent the total of regular cash payments received directly from government, without any requirements to provide goods or services in return for the payment, by all members of the income unit. 'Direct taxes' represent, for Australian income units, the total personal income tax and health insurance levy of all members of the income unit; and, for Swedish income units, all 'negative transfers' as defined in Swedish Income Distribution Survey publications (over 98 per cent of negative transfers in 1984 were direct taxes paid to central and local governments). The direct tax for Australian income units was modelled in the same way as in ABS Household Expenditure Survey 1984: The Effects of Government Benefits and Taxes on Household Income: see discussion on pages 2, 79 and 83 of that publication.
} 
composition, income data in some of the tables are presented in terms of averages per consumption unit (CU). An income unit consisting of a married couple only is taken as $1 \mathrm{CU}$, with a single person income unit regarded as equivalent to $0.6 \mathrm{CU}$ and children $0.25 \mathrm{CU} .{ }^{10}$ This yields the following 'equivalence ratios':

\begin{tabular}{|l|l|}
\hline & Consumption unit \\
\hline Single person only & 0.60 \\
\hline Single parent, one child & 0.85 \\
\hline Married couple only & 1.00 \\
\hline Single parent, two children & 1.10 \\
\hline Married couple, one child & 1.25 \\
\hline Married couple, two children & 1.50 \\
\hline Married couple, three children & 1.75 \\
\hline
\end{tabular}

The real values for Australian income units are money values in Australian currency, but with the average weekly values from HES data converted to average annual rates. The values for Swedish income units are converted from Swedish kroner to Australian dollars using OECD estimates of the Purchasing Power Parity (PPP) of the two currencies in relation to private final consumption expenditure. The relative average money incomes of the various income unit categories are therefore expressed in estimated 'real values', and specifically in terms of their relative capacity to purchase goods and services entering into the private final consumption expenditure of households. ${ }^{11}$ The same PPP ratio (Skr7.2 = \$Al) is used for all income unit types and at all income levels. The presentation of all of the money income comparisons in terms of a uniform purchasing power measure does not, of course, affect the relativity between income magnitudes within each of the countries.

10 In Swedish Income Distribution Survey publications, a slightly different consumption unit is used: a single person is taken as 0.95 consumption units, a couple as 1.65 consumption units and children as 0.40 consumption units. Converted to 'couple units', the relevant ratios are: couples $=1.00$ consumption units, single persons 0.576 consumption units and children 0.242 consumption units. The different equivalence ratios used in the published Swedish income distribution tables do not affect the comparisons in Attachment A.

11 Purchasing power parity (PPP) estimates for Australia, Sweden and a number of other countries have been compiled by the OECD from information supplied by national statistical offices (including Australia), and are to be published in National Accounts: Volume 1-Supplement (forthcoming). Summary estimates have been published in ABS, Gross Domestic Product at Purchasing Power Parity in OECD Countries 1985 issued August 1987. The purchasing power parity of private final consumption expenditure in terms of national currency units per US dollars in 1985 was Skr8.88 for Sweden and \$1.24 for Australia, implying that the $\mathrm{Skr} / \$$ A relationship in terms of average OECD prices was 7.16: 1 in that year. Allowing for the (fractionally) higher increase in the implicit price deflator for private final consumption expenditure in Australia in 1985, the implied ratio for 1984 is Skr7.2=\$A1. It should be noted that no allowance is made for the fact that, to the extent that the provision of services by government without charge or at less than full cost (e.g. on health and education functions) differs between the two countries, the 'real value' of income available for expenditure on other goods and services cannot be accurately represented by private final consumption expenditure adjusted by the PPP ratio. 


\section{Main results}

The comparisons in the 28 Australia/Sweden tables in Attachment A cannot be summarised in a few paragraphs. This section provides some illustrations of the way in which the tables can be used to compare the structures of income distribution and redistribution in the two countries.

\section{The aged population}

The most striking difference in the demographic structure of the two countries is the much larger aged population in Sweden. This is brought out in the first column of Table Al. For each 100 income units in the under 65 age group, there were 34 income units in the 65 years and over age group in Sweden and only 19 income units in this group in Australia.

It follows from this much higher proportion of aged income units in Sweden that the aggregate of net cash transfers to the aged population would need to have been almost twice as great in relation to the income of the non-aged population in order to achieve the same relative level of income support per aged income unit in Sweden as in Australia. In fact, Table Al shows that net cash transfers to the aged in Sweden were far greater than this.

Expressed as a proportion of the total private income of non-aged income units, net cash transfers to the 65 years and over aged group amounted to 14.2 per cent in Sweden compared with only 4.5 per cent in Australia (Table A1). Measured against the money income of the working population, net payments for the income support of the aged were thus more than three times greater in Sweden than in Australia.

Although the level of net cash transfers per aged income unit was much higher in Sweden than in Australia, the average money disposable income of aged people in the two countries was virtually the same in terms of the purchasing power parity (PPP) estimates used in this study (Table A27), and the average in each income decile was also quite similar (Table A28). These outcomes reflect two factors: the higher average levels of private income per aged income unit in Australia; and the effects of income tests in reducing or eliminating the entitlement to benefits of aged people with significant private incomes in Australia, but not in Sweden.

\section{Non-employed working age population}

In the case of the working age population, the most marked difference between the two countries was in the proportion of income units in which there was no employed adult. Such units are usually dependent on government benefits for all or most of their income. ${ }^{12}$

12 Not all income units in which there was no employed adult were mainly dependent on government benefits: in Australia there would, for example, have been significant numbers of early retirees with 
Whereas the proportion of aged to working age income units was much higher in Sweden than in Australia, the proportion of 'dependent' income units in the working age group was much higher in Australia than in Sweden. For each 100 income units in the 20-64 age group in which at least one adult was employed, the number of units in which no adult was employed was 27 in Australia and only 12 in Sweden (Table A4). Moreover, nearly 80 per cent of the much smaller population of 'dependent' units in the working age group in Sweden consisted of single persons, whereas most of the 'dependent' units in Australia were couples with or without children and single parents (Table A4).

The proportion of couples without children in the 20-64 age group with neither partner employed was, at 19 per cent, much higher in Australia than in Sweden - five per cent (Table A4). The difference was mainly attributable to a lower average retiring age in Australia: over five-sixths of the non-employed couples without children in the 20-64 age group in Australia were in the 55-64 age group. In Sweden, the universal old-age pension and the earnings-related pension are available to all aged persons who are otherwise eligible, whether they retire or not. ${ }^{13}$

Among income units with children, the proportion in which the sole parent or both parents were not employed was 16 per cent in Australia but less than four per cent in Sweden. The proportion of single parents who were not employed was 64 per cent in Australia compared with only 16 per cent in Sweden, and the proportion of couples with children in which neither partner was in employment was eight per cent in Australia and about one per cent in Sweden.

\section{Comparison of private and disposable income distributions}

The detailed tables provide numerous comparisons of the distributions of the private and disposable incomes of various population groups. This section illustrates one way in which the data can be used. The share of the total income of selected populations of income units with children which is received by the 50 per cent with the lowest incomes is compared for the two countries and, for each country, for private and disposable incomes.

In the case of all couples with children, the income shares for private and disposable incomes were:

13 US Department of Health and Human Services, Social Security Administration, Social Security Programs Throughout the World 1985: 242. 


\begin{tabular}{|l|c|c|}
\hline $\begin{array}{l}\text { Share of income of } 50 \text { per cent of married } \\
\text { couple with children income units with } \\
\text { lowest: }\end{array}$ & Australia \% & Sweden \% \\
\hline Private incomes & 24.1 & 33.4 \\
\hline Disposable incomes & 33.2 & 39.5 \\
\hline Difference & 9.1 & 6.1 \\
\hline
\end{tabular}

Source: Table Al7 in Attachment A.

The table shows that, in Australia, the share of the 'lower half' in disposable incomes was nine percentage points higher than their share of private incomes but it was still fractionally below the share of the 'lower half' in private incomes in Sweden.

The larger increase in the income share between the two income concepts in Australia (nine percentage points) than in Sweden (six percentage points) is entirely attributable to the benefits paid to the substantial numbers of income units in Australia with neither partner employed. For income units with one partner employed the income shares were fairly similar in both countries:

\begin{tabular}{|l|c|c|}
\hline $\begin{array}{l}\text { Share of income of } 50 \text { per cent of married } \\
\text { couple with children, one employed income } \\
\text { units with lowest: }\end{array}$ & Australia \% & Sweden \% \\
\hline Private incomes & 32.4 & 32.9 \\
\hline Disposable incomes & 36.7 & 38.7 \\
\hline Difference & 4.5 & 5.8 \\
\hline
\end{tabular}

Source: Table Al8 in Attachment A.

For 'married couple with children' income units with both partners employedwhich outnumbered one-worker couples in a ratio of more than five to one in Sweden but which were about the same in number as one-worker couples in Australia (Table A4) - the corresponding figures were as follows:

\begin{tabular}{|l|c|c|}
\hline $\begin{array}{l}\text { Share of income of } 50 \text { per cent of married } \\
\text { couple with children, two employed income } \\
\text { units with lowest: }\end{array}$ & Australia \% & Sweden \% \\
\hline Private incomes & 31.4 & 36.0 \\
\hline Disposable incomes & 34.8 & 40.6 \\
\hline Difference & 3.4 & 4.6 \\
\hline
\end{tabular}

Source: Table A19 in Attachment A. 
The tables show that the impact of cash transfer systems in redistributing income between income units cannot be evaluated in isolation from factors such as the employment status of the members of those units. The point emerges even more clearly from similar data relating to single parent income units. For all single parents, the income shares for private and disposable incomes were:

\begin{tabular}{|l|c|c|}
\hline $\begin{array}{l}\text { Share of income of } \mathbf{5 0} \text { per cent of single } \\
\text { parent income units with lowest: }\end{array}$ & Australia \% & Sweden \% \\
\hline Private incomes & 2.0 & 23.8 \\
\hline Disposable incomes & 32.1 & 39.3 \\
\hline Difference & 30.1 & 15.5 \\
\hline
\end{tabular}

Source: Table A23 in Attachment A.

Restricting the comparison to income units in which the single parent is employed - 36 per cent of the total in Australia and 85 per cent in Sweden - the income shares of the 'lower half' were as follows:

\begin{tabular}{|l|c|c|}
\hline $\begin{array}{l}\text { Share of income of } \mathbf{5 0} \text { per cent of employed } \\
\text { single parent income units with lowest: }\end{array}$ & Australia \% & $\begin{array}{c}\text { Sweden } \\
\%\end{array}$ \\
\hline Private incomes & 23.8 & 31.1 \\
\hline Disposable incomes & 34.7 & 40.3 \\
\hline Difference & 10.9 & 9.2 \\
\hline
\end{tabular}

Source: Table A24 in Attachment A.

\section{Taxes and benefits}

Most income units in Sweden paid more in direct taxes, but received more in direct benefits, than corresponding units in Australia. In both countries, however, the net cash transfers (direct taxes paid minus direct benefits received) were equivalent to about 15 per cent of the private income of all income units in the 20-64 years age group (Table A6) and about 20 per cent of the private income of units in the 20-64 age group in which there was at least one employed adult (Table A12).

The similarity in the proportion of net transfers to private income in the aggregate did not extend to particular categories of income units. For example, one-worker couples with children in Sweden received more in direct benefits than they paid in direct taxes, whereas in Australia the direct taxes paid by this group exceeded benefits received by an amount equivalent to about 17 per cent of private income. By contrast, the net cash transfers paid by two-worker couples with children were equivalent to about 21 per cent of private income in both countries (Table A5). 


\section{Money incomes of households in Australia and the United States 1984}

The income survey used for the United States comparison was the March 1985 Current Population Survey (CPS) conducted by the Bureau of the Census. The Census Bureau has combined data from the Annual Housing Survey, the Income Survey Development Program and the Internal Revenue Service, as well as the CPS results to derive estimates of after-tax income which are designed inter alia to 'measure...differences in purchasing power between subgroups of the population.' ${ }^{14}$

The detailed tables in Attachment B present comparable information for households from the Australian HES and from the study of after-tax incomes for 1984 by the US Bureau of the Census. The tables make use of concepts and classifications of income, households, household income deciles, consumption units and real values. These differ in many respects from those used in the Australia/Sweden comparison. They are defined and explained in the next section.

\section{Concepts and classifications}

Two concepts of income are used in the Australia/United States comparisons: 'before tax income' and 'after tax income'. 'Before tax income' is identical to the 'gross income' concept used in HES and other ABS income survey publications i.e. it includes government cash pensions and benefits as well as all other money receipts received regularly by any member of the household. 'After tax income' is similar to the HES concept of 'disposable income', except that the deductions from gross income include property taxes (US) and general rates (Australia) paid on owner-occupied dwellings. The United States estimates of taxes are based on a simulation which allocated to households direct taxes equivalent to 98.5 per cent of the relevant taxes according to independent estimates. ${ }^{15}$

14 All of the estimates used in the tables in Attachment B are derived from US Bureau of the Census AfterTax Money Income Estimates of Households: 1984 (Special Studies, Series P-23, No. 147), except for the estimates for consumption units used to calculate average incomes per consumption unit in the lower half of Table B2. The latter estimates use information about numbers of children in households of different types from other Census Bureau publications relating to the March 1985 CPS.

15 The taxes allocated in the simulation procedure compare with independent estimates of tax liabilities or collections as follows:

\begin{tabular}{|l|l|l|}
\hline & Simulation \$bn & Independent estimate \$bn \\
\hline Federal income tax & 293.9 & 302.1 \\
\hline State income taxes & 66.6 & 61.9 \\
\hline Payroll tax on households & 115.7 & 117.3 \\
\hline Property taxes & 40.8 & 43.4 \\
\hline & $\mathbf{5 1 7 . 0}$ & $\mathbf{5 2 4 . 7}$ \\
\hline
\end{tabular}


The Australian estimates were also based on a simulation, which resulted in 97 per cent of the revenue collected by government being allocated to households.

The comparisons are made for five household types:

1. Married couples with no children under 18;

2. Married couples with children under 18;

3. Female householder, no husband present, with children under 18;

4. Single person households;

5. All other;

and are also made for households in six age groups of the person nominated as 'head', and for households in owned and rented dwellings.

The populations of households (but not of individual types of households) are ranked in ascending order of their before-tax incomes and divided into deciles. ${ }^{16}$ The distributions of before tax and after tax income in gross income deciles are also compared in the form of 'co-efficients of concentration' for both countries.

The population of households of different types is again expressed in terms of the number of consumption units, using the same equivalence ratios as were used in the Swedish comparison.

Estimated real values of the average before-tax and after-tax money incomes are also calculated for 'all households', and for 23 household categories (five household types, six ages of household head, two forms of housing occupancy and 10 before-tax income deciles.)

The real values are calculated by converting United States dollars to Australian dollars using OECD estimates of the purchasing power parity of the two currencies in relation to private final consumption expenditure. The same PPP ratio $(\mathrm{A} \$ 1=84$ US cents, or $\$ \mathrm{US} 1=\mathrm{A} \$ 1.19)$ is used for all household categories. The presentation of the money income estimates in this form does not affect the relativity between average incomes for particular household categories within each of the two countries.

\section{Main results}

The Australia/United States comparisons in Attachment B are much less comprehensive than the Australia/Sweden comparisons in Attachment A. The

16 Deciles are not identified in the US Census Bureau publication; the decile averages in the Attachment B tables have been calculated by interpretation from tables in the source document. They correspond closely with the quintile averages in a table in the introductory text of the source document. 
tables provide some comparable estimates for Australia and the United States relating to all households, classified by type (i.e. household composition), age of head, nature of housing occupancy and gross income decile. A summary table (Table 8) shows direct taxes paid as a percentage of gross income for each of the housing types identified.

Of the five household types distinguished in the estimates in Attachment B, married couples with children had the highest incomes in both countries. When the estimates are expressed on a 'per consumption unit' rather than a 'per household' basis, however, couples without children had much higher incomes in both countries. The average after-tax real income per consumption unit of married couple households without children was 34 per cent higher than for couples with children in the United States, and 25 per cent higher in Australia (Table B2).

\section{Household type}

The proportion of single parent households is much higher in the United States than in Australia. For every 100 married couple households with children, the number of female single parent households was 22 in the United States compared with nine in Australia (Table Bl). The average income of female single parent households is much lower than the average income of all households in both countries. On a 'per consumption unit' basis, the average after-tax income of such households was 51 per cent of the average for all households in the United States and 68 per cent of the average for all households in Australia (Table B2).

\section{Age of household head}

There is a marked difference between Australia and the United States in the relative incomes of the young and the old (Table B4).

Expressed on a 'per person' basis, the average income of Australian households with heads under 25 years of age was higher than for any other age group, both before tax and after tax. In the United States, by contrast, the average income per person of households with heads under 25 years was lower than for any other age group, both before tax and after tax.

On the same 'per person' basis, the average income of Australian households with heads over 65 years of age was second-lowest of the six age groups distinguished in the study; and the average income of United States households in this age group was second-highest of the six age groups. 


\section{Nature of housing occupancy}

In both countries, the average income of renter households was lower than the average income of owner-occupiers. The per capita after-tax income of households renting the dwellings they occupied was six per cent lower than that of owner-occupiers in Australia, and 26 per cent lower in the United States (Table B5).

\section{Household gross income deciles}

When all households were ranked in ascending order of their gross incomes, the share of the 10 per cent of households with the highest gross incomes in total income was about 2.5 percentage points greater in the United States than in Australia. This was true both in 'before tax' and 'after tax' terms (Table B6).

The co-efficient of concentration for after-tax incomes of all households in the United States was the same as the corresponding co-efficient for the before-tax incomes of all households in Australia.

In Australia the average income after tax per person in the highest household gross income decile exceeded that in the lowest decile in the ratio of 3.4 to 1 . The corresponding ratio in the United States was over 10 to 1 (Table B6).

\section{Direct taxes as percentage of income}

Federal income tax took a much greater proportion of gross incomes in Australia than in the United States (20 per cent compared with 12 per cent). There were, however, two significant types of direct taxes in the United States which had no counterpart in Australia: state indirect taxes (three per cent of gross income) and payroll taxes levied on employees and the self-employed (five per cent of gross income). With the addition of these taxes, the overall proportion of gross incomes taken in direct taxes was virtually the same in both countries (Table B7).

The proportion of gross incomes paid in all direct taxes was significantly higher in the United States at lower income levels, but much the same in the higher income deciles: 
Measuring and Promoting Wellbeing: How Important is Economic Growth?

\begin{tabular}{|l|c|c|c|}
\hline & Australia \% & $\begin{array}{c}\text { United States } \\
\%\end{array}$ & $\begin{array}{c}\text { Difference } \\
\%\end{array}$ \\
\hline \multicolumn{4}{|l|}{} \\
\hline Lirect taxes as percentage of gross income in: \\
\hline Lecond decile & 3.4 & 7.1 & +3.7 \\
\hline Third decile & 3.6 & 7.1 & +3.5 \\
\hline Fourth decile & 5.3 & 10.4 & +5.1 \\
\hline Fifth decile & 12.7 & 13.4 & +0.7 \\
\hline Sixth decile & 16.1 & 15.7 & -0.4 \\
\hline Seventh decile & 18.7 & 18.2 & -0.5 \\
\hline Eighth decile & 21.0 & 19.7 & -1.3 \\
\hline Ninth decile & 22.4 & 21.7 & -0.7 \\
\hline Highest 10\% & 23.9 & 23.7 & -0.2 \\
\hline Overall average & 29.1 & 28.7 & -0.4 \\
\hline
\end{tabular}

Source: Table B8 in Attachment B.

The similarity between the two countries in the proportion of gross income taken in direct taxes in the six highest gross income deciles was also reflected in similar percentages of gross income paid in direct taxes for most household types, for most age groups of household head and for households renting or owning the dwelling they occupied (Table B8).

Because average real household incomes are significantly higher in the United States than in Australia, the similarity in the ratios of direct taxes to gross incomes at given levels of income defined in relation to the structure of incomes within each country is not inconsistent with Australian tax/income ratios which were higher at given levels of income defined in relation to their purchasing power. The following table shows that, on the latter basis, direct tax rates were two to four percentage points higher in Australia over a wide range of household incomes:

\begin{tabular}{|l|c|c|c|}
\hline & Australia $\%$ & $\begin{array}{c}\text { United States } \\
\%\end{array}$ & $\begin{array}{c}\text { Difference } \\
\%\end{array}$ \\
\hline \multicolumn{3}{|c|}{ Direct taxes as percentage of gross income, at real gross income of: } \\
\hline \$A15,000 & 13.2 & 11.2 & -2.0 \\
\hline \$A20,000 & 17.3 & 14.0 & -3.3 \\
\hline \$A25,000 & 20.1 & 16.2 & -3.9 \\
\hline \$A30,000 & 21.8 & 18.3 & -3.5 \\
\hline \$A35,000 & 23.0 & 19.4 & -3.6 \\
\hline \$A40,000 & 24.1 & 20.6 & -3.5 \\
\hline
\end{tabular}

Source: Derived by interpolation from Tables B6 and B7 in Attachment B. 


\section{Concluding comments}

This paper has shown that there are very substantial differences between Australia, Sweden and the United States in the distribution of household money incomes. Estimates of the relative per capita real income levels for entire populations may not reveal much about the real household incomes of particular groups within a country. There are major differences between countries in the degree of concentration or dispersion of household incomes, both in the aggregate and for groups of households which are relatively homogeneous in terms of family composition and the employment status of household members. Differing structures of cash transfers have an important impact on the distribution of disposable incomes, but differences in the degree of dispersion of private incomes appear to have an even larger influence. Whilst analysis of the distribution of income of all households can go some distance towards identifying the key features of the structure of household incomes, there is no substitute for detailed comparisons at the level of disaggregation undertaken in the Australia/Sweden study reported in this paper.

\section{Attachment A}

\section{Australia and Sweden 1984: List of tables}

A: Distribution of total household income among households: period income from 1981-82 Income and Housing Survey and current income from 1984 Household Expenditure Survey

\section{All income units}

Al: Number, average real private and disposable income, and total real private and disposable income, by age groups

A2: Persons and employed persons per 100 income units, by private income quantiles

A3: Average real income by income quantiles:

(a) Private income, units ranked by private income

(b) Disposable income, units ranked by private income

(c) disposable income, units ranked by disposable income 
Measuring and Promoting Wellbeing: How Important is Economic Growth?

Income units in 20-64 age group

A4: Number, by income unit type and employment status

A5: Average real private and disposable income, by income unit type and employment status

A6: Average real private income, cash transfers and disposable income, by income unit type

A7: Average real disposable income, by disposable income quantile

\section{With no employed adult}

A8: Average real income by income quantiles:

(a) Private income, units ranked by private income unit

(b) Disposable income, units ranked by disposable income unit

With at least one employed adult

A9: Persons, employed persons and consumption units per 100 income units, by private income quantiles:
(a) Married couples without children
(b) Married couples with children
(c) Single parents
(d) Single persons
(e) All income units

A10 Average real private income, by private income quantiles:
(a) Married couples without children
(b) Married couples with children
(c) Single parents
(d) Single persons
(e) All income units

A11: Average real disposable income, by private income quantiles:
(a) Married couples without children
(b) Married couples with children
(c) Single parents 
(d) Single persons

(e) All income units

A12: Average real private and disposable income per consumption unit, by private income quantile:

(a) Married couples without children

(b) Married couples with children

(c) Single parents

(d) Single persons

A13: Average disposable income as percentage of average private income, by income unit type and private income quantile

A14: Average real income per consumption unit, (CU) by income unit type and private income quantile, Sweden as \% of Australia:

(a) Average real private income per $\mathrm{CU}$

(b) Average real disposable income per $\mathrm{CU}$

\section{Married couple income units without children}

A15: One employed: average real income by income quantiles:

(a) Private income, units ranked by private income

(b) Disposable income, units ranked by disposable income

A16: Both employed: average real income by income quantiles:

(a) Private income, units ranked by private income

(b) Disposable income, units ranked by disposable income

A17: All units: average real income by income quantiles:

(a) Private income, units ranked by private income

(b) Disposable income, units ranked by disposable income

A18: One employed: average real income by income quantiles:

(a) Private income, units ranked by private income

(b) Disposable income, units ranked by disposable income

A19: Both employed: average real income by income quantiles

(a) Private income, units ranked by private income 
Measuring and Promoting Wellbeing: How Important is Economic Growth?

(b) Disposable income, units ranked by disposable income A20: One child: average real income by income quantiles:

(a) Private income, units ranked by private income

(b) Disposable income, units ranked by disposable income A21: Two childen: average real income by income quantiles:

(a) Private income, units ranked by private income

(b) Disposable income, units ranked by disposable income A22: Three children: average real income by income quantiles:

(a) Private income, units ranked by private income

(b) Disposable income, units ranked by disposable income Single parent income units

A23: All units: average real income by income quantiles:

(a) Private income, units ranked by private income

(b) Disposable income, units ranked by disposable income

A24: Parent employed: average real income by income quantiles:

(a) Private income, units ranked by private income

(b) Disposable income, units ranked by disposable income Single person income units

A25: Person employed: average real income by income quantiles:

(a) Private income, units ranked by private income

(b) Disposable income, units ranked by disposable income Income units in 18-19 years of age group A26: All units: Average real income by income quantiles

(a) Private income, units ranked by private income

(b) Disposable income, units ranked by disposable income Income units in $65+$ age group

A27: All units: average real private income, cash transfers and disposable income A28: All units, average real income by income quintiles: 
(a) Private income, units ranked by private income

(b) Disposable income, units ranked by disposable income

\section{Definitions of terms}

A29: Terms used in Australia/Sweden comparisons

Table A Distribution of total household income among households: period income from 1981-82 Income and Housing Survey and current income from 1984 Household Expenditure Survey

\begin{tabular}{|c|c|c|c|c|}
\hline \multirow[b]{2}{*}{$\begin{array}{l}\% \text { of total } \\
\text { household } \\
\text { income }\end{array}$} & \multicolumn{2}{|c|}{$\%$ of households } & \multicolumn{2}{|c|}{ Cumulative $\%$ of households } \\
\hline & $\begin{array}{c}1981 / 82 \\
\text { Income and } \\
\text { Housing Survey }\end{array}$ & 1984 HES & $\begin{array}{c}1981 / 82 \\
\text { Income and } \\
\text { Housing } \\
\text { Survey }\end{array}$ & 1984 HES \\
\hline 5 & 20.6 & 19.6 & 20.6 & 19.6 \\
\hline 10 & 10.7 & 11.2 & 31.3 & 30.9 \\
\hline 15 & 7.7 & 8.1 & 39.0 & 39.0 \\
\hline 20 & 6.6 & 6.8 & 45.6 & 45.8 \\
\hline 25 & 5.8 & 6.0 & 51.4 & 51.8 \\
\hline 30 & 5.3 & 5.4 & 56.7 & 57.2 \\
\hline 35 & 4.9 & 4.8 & 61.5 & 62.0 \\
\hline 40 & 4.5 & 4.4 & 66.0 & 66.4 \\
\hline 45 & 4.2 & 4.1 & 70.2 & 70.6 \\
\hline 50 & 3.9 & 3.8 & 74.1 & 74.4 \\
\hline 55 & 3.6 & 3.6 & 77.1 & 78.0 \\
\hline 60 & 3.4 & 3.4 & 81.1 & 81.4 \\
\hline 65 & 3.2 & 3.2 & 84.3 & 84.5 \\
\hline 70 & 3.0 & 2.9 & 87.3 & 87.5 \\
\hline 75 & 2.8 & 2.7 & 90.0 & 90.2 \\
\hline 80 & 2.5 & 2.5 & 92.6 & 92.7 \\
\hline 85 & 2.3 & 2.3 & 94.9 & 95.0 \\
\hline 90 & 2.1 & 2.0 & 97.0 & 97.0 \\
\hline 95 & 1.8 & 1.7 & 98.8 & 98.7 \\
\hline 100 & 1.2 & 1.3 & 100.0 & 100.0 \\
\hline
\end{tabular}


Table A1 All income units: number, average real private and disposable income, and total real private and disposable income, by age groups

\begin{tabular}{|c|c|c|c|c|c|}
\hline & $\begin{array}{c}\text { Number } \\
\text { of income } \\
\text { units } \\
\text { ('000) }\end{array}$ & $\begin{array}{c}\text { Average } \\
\text { private } \\
\text { income } \\
\text { (\$A) }\end{array}$ & $\begin{array}{c}\text { Average } \\
\text { disposable } \\
\text { income } \\
\text { (\$A) }\end{array}$ & $\begin{array}{l}\text { Total } \\
\text { private } \\
\text { income } \\
\text { (\$Am) }\end{array}$ & $\begin{array}{c}\text { Total } \\
\text { disposable } \\
\text { income } \\
\text { (\$Am) }\end{array}$ \\
\hline \multicolumn{6}{|c|}{ Income units in: } \\
\hline \multicolumn{6}{|c|}{ 20-64 age group } \\
\hline Australia & $5,115.7$ & 19,170 & 16,297 & 98,068 & 83,371 \\
\hline Sweden & $3,131.8$ & 15,501 & 13,029 & 48,546 & 40,804 \\
\hline \multicolumn{6}{|c|}{$18-19$ age group } \\
\hline Australia & 435.9 & 6,299 & 5,999 & 2,746 & 2,615 \\
\hline Sweden & 230.0 & 3,556 & 3,278 & 818 & 754 \\
\hline \multicolumn{6}{|c|}{ All under 65 age group } \\
\hline Australia & $5,551.6$ & 18,159 & 15,489 & 100,814 & 85,986 \\
\hline Sweden & $3,361.8$ & 14,684 & 12,362 & 49,364 & 41,558 \\
\hline \multicolumn{6}{|c|}{$65+$ age group } \\
\hline Australia & $1,039.2$ & 4,061 & 8,473 & 4,220 & 8,805 \\
\hline Sweden & $1,141.0$ & 2,056 & 8,209 & 2,346 & 9,366 \\
\hline \multicolumn{6}{|c|}{ Total } \\
\hline Australia & $6,590.8$ & 15,936 & 14,382 & 105,034 & 94,791 \\
\hline Sweden & $4,502.8$ & 11,484 & 11,309 & 51,710 & 50,924 \\
\hline
\end{tabular}

Table A2 All income units; persons and employed persons per 100 income units, by private income quantiles

\begin{tabular}{|l|c|c|c|c|}
\hline \multicolumn{5}{|c|}{ Number per 100 income units } \\
\hline \multirow{2}{*}{$\begin{array}{l}\text { Private income } \\
\text { quantile }\end{array}$} & \multicolumn{2}{|c|}{ Persons } & \multicolumn{2}{c|}{ Employed persons } \\
\cline { 2 - 5 } & Australia & Sweden & Australia & Sweden \\
\hline Lowest 5\% & 175 & 123 & 6 & 5 \\
\hline Lowest 10\% & 171 & 127 & 6 & 5 \\
\hline Second decile & 157 & 130 & 7 & 3 \\
\hline Third decile & 167 & 134 & 20 & 6 \\
\hline Fourth decile & 175 & 132 & 72 & 62 \\
\hline Fifth decile & 176 & 148 & 99 & 100 \\
\hline Sixth decile & 214 & 156 & 105 & 108 \\
\hline Seventh decile & 232 & 179 & 113 & 114 \\
\hline Eighth decile & 279 & 252 & 131 & 152 \\
\hline Ninth decile & 292 & 287 & 158 & 185 \\
\hline Highest 10\% & 308 & 290 & 173 & 193 \\
\hline Highest 5\% & 315 & 294 & 175 & 191 \\
\hline Highest 2.5\% & 307 & 290 & 168 & 189 \\
\hline All income units & 217 & 183 & 88 & 93 \\
\hline
\end{tabular}


Table A3 All income units: average real private and disposable income by income quantiles

\begin{tabular}{|c|c|c|c|c|}
\hline \multicolumn{5}{|c|}{ Average annual income } \\
\hline \multirow{2}{*}{ Income quantile } & \multicolumn{2}{|c|}{$\$ A^{\prime} 000$} & \multicolumn{2}{|c|}{ As \% of total } \\
\hline & Australia & Sweden & Australia & Sweden \\
\hline \multicolumn{5}{|c|}{ (a) Private income, units ranked by private income } \\
\hline Lowest $5 \%$ & 0.0 & -0.4 & 0.0 & -0.2 \\
\hline Lowest $10 \%$ & 0.0 & -0.2 & 0.0 & -0.1 \\
\hline Second decile & 0.1 & 0.4 & 0.1 & 0.4 \\
\hline Third decile & 1.4 & 1.5 & 0.9 & 1.3 \\
\hline Fourth decile & 6.9 & 3.8 & 4.3 & 3.3 \\
\hline Fifth decile & 12.5 & 7.4 & 7.9 & 6.4 \\
\hline Sixth decile & 15.8 & 11.1 & 9.9 & 9.7 \\
\hline Seventh decile & 19.3 & 13.9 & 12.1 & 12.1 \\
\hline Eighth decile & 24.2 & 17.9 & 15.2 & 15.5 \\
\hline Ninth decile & 30.9 & 23.6 & 19.3 & 20.5 \\
\hline Highest $10 \%$ & 48.4 & 35.4 & 30.3 & 30.9 \\
\hline Highest $5 \%$ & 58.0 & 41.9 & 18.1 & 18.2 \\
\hline Highest $2.5 \%$ & 68.7 & 49.1 & 10.8 & 10.7 \\
\hline All income units & 16.0 & 11.5 & 100.0 & 100.0 \\
\hline Co-efficient of concentration & 0.504 & 0.520 & 0.504 & 0.520 \\
\hline \multicolumn{5}{|c|}{ (b) Disposable income, units ranked by private income } \\
\hline Lowest $5 \%$ & 5.4 & 5.1 & 1.9 & 2.2 \\
\hline Lowest $10 \%$ & 5.2 & 5.9 & 3.6 & 5.2 \\
\hline Second decile & 5.3 & 6.9 & 3.7 & 6.1 \\
\hline Third decile & 6.9 & 6.9 & 4.8 & 6.1 \\
\hline Fourth decile & 8.2 & 7.0 & 5.7 & 6.2 \\
\hline Fifth decile & 11.1 & 8.8 & 7.7 & 7.7 \\
\hline Sixth decile & 13.4 & 9.8 & 9.3 & 8.6 \\
\hline Seventh decile & 16.0 & 11.3 & 11.1 & 10.0 \\
\hline Eighth decile & 19.5 & 14.7 & 13.5 & 13.0 \\
\hline Ninth decile & 24.1 & 18.0 & 16.7 & 15.9 \\
\hline Highest $10 \%$ & 34.4 & 23.8 & 23.9 & 21.2 \\
\hline Highest $5 \%$ & 39.5 & 26.9 & 13.7 & 11.9 \\
\hline Highest $2.5 \%$ & 44.4 & 30.2 & 7.7 & 6.7 \\
\hline All income units & 14.4 & 11.3 & 100.0 & 100.0 \\
\hline Co-efficient of concentration & 0.335 & 0.259 & 0.335 & 0.259 \\
\hline \multicolumn{5}{|c|}{ (c) Disposable income, units ranked by disposable income } \\
\hline Lowest $5 \%$ & 1.3 & 1.5 & 0.4 & 0.7 \\
\hline Lowest $10 \%$ & 2.7 & 2.8 & 1.9 & 2.5 \\
\hline Second decile & 5.2 & 5.3 & 3.6 & 4.7 \\
\hline Third decile & 7.4 & 6.5 & 5.1 & 5.7 \\
\hline Fourth decile & 9.3 & 7.7 & 6.5 & 6.8 \\
\hline Fifth decile & 11.4 & 8.9 & 7.9 & 7.9 \\
\hline Sixth decile & 13.6 & 10.4 & 9.4 & 9.2 \\
\hline Seventh decile & 16.1 & 12.7 & 11.2 & 11.2 \\
\hline Eighth decile & 19.5 & 15.6 & 13.6 & 13.8 \\
\hline Ninth decile & 24.1 & 18.5 & 16.8 & 16.4 \\
\hline Highest $10 \%$ & 34.5 & 24.7 & 24.0 & 21.9 \\
\hline Highest $5 \%$ & 39.7 & 27.9 & 13.8 & 12.3 \\
\hline Highest $2.5 \%$ & 44.9 & 31.6 & 7.8 & 7.0 \\
\hline All income units & 14.4 & 11.3 & 100.0 & 100.0 \\
\hline Co-efficient of concentration & 0.349 & 0.311 & 0.349 & 0.311 \\
\hline
\end{tabular}


Measuring and Promoting Wellbeing: How Important is Economic Growth?

Table A4 number of income units in 20-64 age group, by income unit type and employment status

\begin{tabular}{|c|c|c|c|c|c|c|}
\hline & \multicolumn{2}{|c|}{ Number of income units } & \multicolumn{2}{|c|}{$\begin{array}{c}\% \text { of income unit } \\
\text { type }\end{array}$} & \multicolumn{2}{|c|}{$\begin{array}{c}\% \text { of all income } \\
\text { units } 20-64 \text { years }\end{array}$} \\
\hline & $\begin{array}{c}\text { Australia } \\
\text { ('000) }\end{array}$ & $\begin{array}{c}\text { Sweden } \\
\text { ('000) }\end{array}$ & $\begin{array}{c}\text { Australia } \\
\%\end{array}$ & $\begin{array}{c}\text { Sweden } \\
\%\end{array}$ & $\begin{array}{c}\text { Australia } \\
\%\end{array}$ & $\begin{array}{c}\text { Sweden } \\
\%\end{array}$ \\
\hline \multicolumn{7}{|c|}{ Married couple without children } \\
\hline 0 employed & 210.1 & 33.2 & 18.8 & 4.9 & 4.11 & 1.06 \\
\hline 1 employed & 347.3 & 169.9 & 31.1 & 25.1 & 6.79 & 5.42 \\
\hline 2 employed & 559.7 & 474.4 & 50.1 & 70.0 & 10.94 & 15.15 \\
\hline Total & $1,117.1$ & 677.5 & 100.0 & 100.0 & 21.84 & 21.63 \\
\hline $\begin{array}{l}\text { Of which } 1+ \\
\text { employed }\end{array}$ & 907.0 & 644.3 & 81.2 & 95.1 & 17.73 & 20.57 \\
\hline \multicolumn{7}{|c|}{ Married couple with children } \\
\hline 0 employed & 151.3 & 10.7 & 8.2 & 1.2 & 2.96 & 0.34 \\
\hline 1 employed & 844.5 & 137.9 & 46.0 & 16.1 & 16.51 & 4.40 \\
\hline 2 employed & 841.9 & 708.3 & 45.8 & 82.7 & 16.46 & 22.61 \\
\hline Total & $1,837.7$ & 856.9 & 100.0 & 100.0 & 35.92 & 27.36 \\
\hline $\begin{array}{l}\text { Of which } 1+ \\
\text { employed }\end{array}$ & $1,686.5$ & 846.2 & 91.8 & 98.8 & 32.97 & 27.02 \\
\hline \multicolumn{7}{|c|}{ Single parent } \\
\hline $\begin{array}{l}\text { Not } \\
\text { employed }\end{array}$ & 189.5 & 29.8 & 63.7 & 14.9 & 3.70 & 0.95 \\
\hline Employed & 108.2 & 169.6 & 36.3 & 85.1 & 2.12 & 5.42 \\
\hline Total & 297.7 & 199.4 & 100.0 & 100.0 & 5.82 & 6.37 \\
\hline \multicolumn{7}{|c|}{ Single person } \\
\hline $\begin{array}{l}\text { Not } \\
\text { employed }\end{array}$ & 522.7 & 264.4 & 28.1 & 18.9 & 10.22 & 8.44 \\
\hline Employed & $1,340.4$ & $1,133.6$ & 71.9 & 81.1 & 26.20 & 36.20 \\
\hline Total & $1,863.1$ & $1,398.0$ & 100.0 & 100.0 & 36.42 & 44.64 \\
\hline \multicolumn{7}{|c|}{ All income units 20-64 } \\
\hline 0 employed & $1,973.6$ & 338.1 & 21.0 & 10.8 & 20.99 & 10.80 \\
\hline $\begin{array}{l}1+ \\
\text { employed }\end{array}$ & $4,042.1$ & $2,793.7$ & 79.0 & 89.2 & 79.01 & 89.20 \\
\hline Total & $5,115.7$ & $3,131.8$ & 100.0 & 100.0 & 100.0 & 100.0 \\
\hline
\end{tabular}


Table A5 Average real private and disposable income of income units in 20-64 age group by income unit type and employment status

\begin{tabular}{|c|c|c|c|c|c|c|}
\hline & \multicolumn{3}{|c|}{ Private income } & \multicolumn{3}{|c|}{ Disposable income } \\
\hline & $\begin{array}{c}\text { Australia } \\
\text { \$A }\end{array}$ & $\begin{array}{c}\text { Sweden } \\
\text { \$A }\end{array}$ & $\begin{array}{c}\mathbf{A} / \mathbf{S} \\
\%\end{array}$ & $\begin{array}{c}\text { Australia } \\
\text { \$A }\end{array}$ & $\begin{array}{c}\text { Sweden } \\
\text { \$A }\end{array}$ & $\begin{array}{c}\text { A/S } \\
\%\end{array}$ \\
\hline \multicolumn{7}{|c|}{ Married couple without children } \\
\hline 0 Employed & 4,594 & 1,278 & 359.4 & 9,896 & 11,431 & 86.6 \\
\hline 1 employed & 22,120 & 13,751 & 160.9 & 17,504 & 14,057 & 124.5 \\
\hline 2 employed & 33,484 & 25,474 & 131.4 & 25,609 & 18,293 & 140.0 \\
\hline Total & 24,519 & 21,349 & 114.8 & 20,135 & 16,890 & 119.2 \\
\hline $\begin{array}{l}\text { Of which } 1+ \\
\text { employed }\end{array}$ & 29,133 & 22,376 & 130.2 & 22,506 & 17,182 & 131.0 \\
\hline \multicolumn{7}{|c|}{ Married couple with children } \\
\hline 0 employed & 2,337 & 2,986 & 78.3 & 10,585 & 11,418 & 92.7 \\
\hline 1 employed & 22,616 & 14,598 & 154.9 & 18,672 & 14,821 & 126.0 \\
\hline 2 employed & 31,396 & 24,766 & 126.8 & 24,764 & 19,627 & 126.2 \\
\hline Total & 24,969 & 22,863 & 109.2 & 20,797 & 18,751 & 110.9 \\
\hline $\begin{array}{l}\text { Of which } 1+ \\
\text { employed }\end{array}$ & 26,999 & 23,113 & 116.8 & 21,713 & 18,849 & 115.2 \\
\hline \multicolumn{7}{|c|}{ Single parent } \\
\hline Not employed & 1,297 & 986 & 131.5 & 7,433 & 9,737 & 76.3 \\
\hline Employed & 15,004 & 10,251 & 146.4 & 14,100 & 11,959 & 117.9 \\
\hline Total & 6,280 & 8,876 & 70.8 & 9,857 & 11,626 & 84.8 \\
\hline \multicolumn{7}{|c|}{ Single person } \\
\hline Not employed & 1,448 & 1,056 & 137.1 & 4,843 & 5,459 & 112.7 \\
\hline Employed & 16,535 & 10,973 & 150.7 & 12,825 & 8,403 & 152.6 \\
\hline Total & 12,303 & 9,098 & 135.2 & 10,586 & 7,848 & 134.9 \\
\hline \multicolumn{7}{|c|}{ All income units $20-64$} \\
\hline 0 employed & 2,162 & 1,125 & 192.2 & 7,098 & 6,612 & 107.4 \\
\hline $1+$ employed & 23,687 & 17,237 & 137.4 & 18,740 & 13,807 & 135.7 \\
\hline Total & 19,170 & 15,501 & 123.7 & 16,297 & 13,029 & 125.1 \\
\hline
\end{tabular}


Table A6 Average real private income, cash transfers and disposable income of income units in 20-64 age group, by income unit type

\begin{tabular}{|c|c|c|c|c|c|c|}
\hline & $\begin{array}{l}\text { Private } \\
\text { income }\end{array}$ & $\begin{array}{c}\text { Direct } \\
\text { benefits } \\
\text { received }\end{array}$ & $\begin{array}{c}\text { Direct } \\
\text { taxes } \\
\text { paid }\end{array}$ & $\begin{array}{l}\text { Net cash } \\
\text { transfers }\end{array}$ & $\begin{array}{l}\text { Disposable } \\
\text { income }\end{array}$ & $\begin{array}{c}\text { Disposable } \\
\text { income } \\
\text { as \% of } \\
\text { private } \\
\text { income }\end{array}$ \\
\hline & \multicolumn{5}{|c|}{ Average annual value (\$A) } & $\%$ \\
\hline \multicolumn{7}{|c|}{ Married couple without children } \\
\hline Australia & 24,519 & $+1,336$ & $-5,720$ & $-4,384$ & 20,135 & 82.1 \\
\hline Sweden & 21,349 & $+3,986$ & $-8,445$ & $-4,459$ & 16,890 & 79.1 \\
\hline $\begin{array}{l}\text { Australia as \% } \\
\text { of Sweden }\end{array}$ & 114.8 & & & & 119.2 & \\
\hline \multicolumn{7}{|c|}{ Married couple with children } \\
\hline Australia & 24,968 & $+1,576$ & $-5,748$ & $-4,172$ & 20,796 & 83.3 \\
\hline Sweden & 22,863 & $+3,611$ & $-7,723$ & $-4,112$ & 18,751 & 82.0 \\
\hline $\begin{array}{l}\text { Australia as \% } \\
\text { of Sweden }\end{array}$ & 109.2 & & & & 110.9 & \\
\hline \multicolumn{7}{|c|}{ Single parents } \\
\hline Australia & 6,280 & $+4,809$ & $-1,232$ & $+3,577$ & 9,857 & 157.0 \\
\hline Sweden & 8,876 & $+5,778$ & $-3,028$ & $+2,750$ & 11,626 & 131.0 \\
\hline $\begin{array}{l}\text { Australia as \% } \\
\text { of Sweden }\end{array}$ & 70.8 & & & & 84.8 & \\
\hline \multicolumn{7}{|c|}{ Single persons without children } \\
\hline Australia & 12,303 & $+1,124$ & $-2,841$ & $-1,717$ & 10,586 & 86.0 \\
\hline Sweden & 9,098 & $+2,472$ & $-3,722$ & $-1,250$ & 7,848 & 86.3 \\
\hline $\begin{array}{l}\text { Australia as \% } \\
\text { of Sweden }\end{array}$ & 135.2 & & & & 134.9 & \\
\hline \multicolumn{7}{|c|}{ All income units in $20-64$ age group } \\
\hline Australia & 19,170 & $+1,547$ & $-4,420$ & $-2,873$ & 16,297 & 85.0 \\
\hline Sweden & 15,501 & $+3,320$ & $-5,792$ & $-2,472$ & 13,029 & 84.1 \\
\hline $\begin{array}{l}\text { Australia as \% } \\
\text { of Sweden }\end{array}$ & 123.7 & & & & 125.1 & \\
\hline
\end{tabular}


Table A7 All income units in 20-64 age group: average real disposable income, by disposable income quantile

\begin{tabular}{|l|c|c|c|c|}
\hline & \multicolumn{4}{|c|}{ Average disposable income } \\
\hline $\begin{array}{l}\text { Disposable income } \\
\text { quantile }\end{array}$ & $\begin{array}{c}\text { Australia } \\
\text { (\$A'000) }\end{array}$ & $\begin{array}{c}\text { Sweden } \\
\text { (\$A'000) }\end{array}$ & $\begin{array}{c}\text { Australia as } \\
\text { \% of total }\end{array}$ & $\begin{array}{c}\text { Sweden as } \\
\text { \% of total }\end{array}$ \\
\hline Lowest 5\% & 2.1 & 2.4 & 0.6 & 0.9 \\
\hline Lowest 10\% & 3.3 & 3.8 & 2.0 & 2.9 \\
\hline Second decile & 6.7 & 6.8 & 4.1 & 5.2 \\
\hline Third decile & 9.6 & 8.0 & 5.9 & 6.1 \\
\hline Fourth decile & 11.8 & 9.1 & 7.2 & 7.0 \\
\hline Fifth decile & 13.7 & 10.7 & 8.4 & 8.2 \\
\hline Sixth decile & 15.8 & 13.0 & 9.7 & 10.0 \\
\hline Seventh decile & 18.4 & 15.4 & 11.3 & 11.8 \\
\hline Eighth decile & 21.7 & 17.6 & 13.3 & 13.5 \\
\hline Ninth decile & 25.9 & 20.0 & 15.9 & 15.4 \\
\hline Highest 10\% & 36.1 & 25.9 & 22.2 & 19.9 \\
\hline Highest 5\% & 41.3 & 29.0 & 12.7 & 11.1 \\
\hline Highest 2.5\% & 46.6 & 32.6 & 7.2 & 6.2 \\
\hline All income units & 16.3 & 13.0 & 100.0 & 100.0 \\
\hline Co-efficient of concentration & 0.314 & 0.278 & 0.314 & 0.278 \\
\hline
\end{tabular}


Measuring and Promoting Wellbeing: How Important is Economic Growth?

Table A8 Income units aged 20-64 with no employed adult: average real private and disposable income by income quantiles

\begin{tabular}{|c|c|c|c|c|}
\hline \multicolumn{5}{|c|}{ Average annual income } \\
\hline Income quantile & $\begin{array}{c}\text { Australia } \\
\$ A^{\prime} 000\end{array}$ & $\begin{array}{l}\text { Sweden } \\
\$ A^{\prime} 000\end{array}$ & $\begin{array}{c}\text { Australia as } \\
\% \text { of total }\end{array}$ & $\begin{array}{l}\text { Sweden as } \\
\% \text { of total }\end{array}$ \\
\hline \multicolumn{5}{|c|}{ (a) Private income, units ranked by private income } \\
\hline Lowest $10 \%$ & 0.0 & -0.1 & 0.0 & -1.0 \\
\hline Second decile & 0.0 & 0.0 & 0.0 & 0.0 \\
\hline Third decile & 0.0 & 0.0 & 0.0 & 0.1 \\
\hline Fourth decile & 0.0 & 0.1 & 0.0 & 1.0 \\
\hline Fifth decile & 0.0 & 0.3 & 0.0 & 2.7 \\
\hline Sixth decile & 0.1 & 0.8 & 0.6 & 6.8 \\
\hline Seventh decile & 0.6 & 1.3 & 2.9 & 11.2 \\
\hline Eighth decile & 1.7 & 1.7 & 7.9 & 15.3 \\
\hline Ninth decile & 4.9 & 2.3 & 22.4 & 20.9 \\
\hline Highest $10 \%$ & 14.4 & 4.8 & 66.2 & 43.0 \\
\hline Highest $5 \%$ & 19.1 & 6.6 & 44.2 & 59.0 \\
\hline All income units & 2.2 & 1.1 & 100.0 & 100.0 \\
\hline $\begin{array}{l}\text { Co-efficient of } \\
\text { concentration }\end{array}$ & 0.802 & 0.638 & 0.802 & 0.638 \\
\hline \multicolumn{5}{|c|}{ (b) Disposable income, units ranked by disposable income } \\
\hline Lowest $10 \%$ & 1.5 & 1.1 & 2.1 & 1.6 \\
\hline Second decile & 4.0 & 2.6 & 5.6 & 3.9 \\
\hline Third decile & 4.5 & 4.3 & 6.3 & 6.4 \\
\hline Fourth decile & 5.0 & 5.4 & 7.0 & 8.2 \\
\hline Fifth decile & 5.9 & 6.1 & 8.3 & 9.2 \\
\hline Sixth decile & 7.0 & 6.5 & 9.9 & 9.9 \\
\hline Seventh decile & 8.0 & 7.3 & 11.3 & 11.1 \\
\hline Eighth decile & 9.0 & 8.3 & 12.7 & 12.6 \\
\hline Ninth decile & 10.6 & 10.3 & 14.9 & 15.5 \\
\hline Highest $10 \%$ & 15.5 & 14.3 & 21.9 & 21.6 \\
\hline Highest 5\% & 18.2 & 16.2 & 12.8 & 12.2 \\
\hline All income units & 7.1 & 6.6 & 100.0 & 100.0 \\
\hline $\begin{array}{l}\text { Co-efficient of } \\
\text { concentration }\end{array}$ & 0.290 & 0.302 & 0.290 & 0.302 \\
\hline
\end{tabular}


Table A9 Income units in 20-64 age group with at least one employed adult: persons, employed persons and consumption units per 100 income units, by income unit type and private income quantile

\begin{tabular}{|c|c|c|c|c|c|c|}
\hline \multicolumn{7}{|c|}{ Number per 100 income units } \\
\hline \multirow{2}{*}{ Private income } & \multicolumn{2}{|c|}{ Persons } & \multicolumn{2}{|c|}{ Employed persons } & \multicolumn{2}{|c|}{ Consumption units } \\
\hline & Australia & Sweden & Australia & Sweden & Australia & Sweden \\
\hline \multicolumn{7}{|c|}{ (a) Married couples without children } \\
\hline Lowest $5 \%$ & 200 & 200 & 138 & 123 & 100 & 100 \\
\hline Lowest $10 \%$ & 200 & 200 & 137 & 124 & 100 & 100 \\
\hline Second decile & 200 & 200 & 122 & 133 & 100 & 100 \\
\hline Third decile & 200 & 200 & 133 & 149 & 100 & 100 \\
\hline Fourth decile & 200 & 200 & 141 & 165 & 100 & 100 \\
\hline Fifth decile & 200 & 200 & 164 & 184 & 100 & 100 \\
\hline Sixth decile & 200 & 200 & 177 & 194 & 100 & 100 \\
\hline Seventh decile & 200 & 200 & 186 & 196 & 100 & 100 \\
\hline Eighth decile & 200 & 200 & 187 & 199 & 100 & 100 \\
\hline Ninth decile & 200 & 200 & 188 & 198 & 100 & 100 \\
\hline Highest $10 \%$ & 200 & 200 & 182 & 190 & 100 & 100 \\
\hline Highest 5\% & 200 & 200 & 181 & 188 & 100 & 100 \\
\hline Highest $2.5 \%$ & 200 & 200 & 171 & 186 & 100 & 100 \\
\hline All income units & 200 & 200 & 162 & 174 & 100 & 100 \\
\hline \multicolumn{7}{|c|}{ (b) Married couples with children } \\
\hline Lowest $5 \%$ & 404 & 363 & 146 & 142 & 151 & 141 \\
\hline Lowest $10 \%$ & 401 & 385 & 144 & 149 & 150 & 146 \\
\hline Second decile & 417 & 390 & 128 & 151 & 154 & 147 \\
\hline Third decile & 408 & 389 & 121 & 171 & 152 & 147 \\
\hline Fourth decile & 397 & 383 & 134 & 187 & 149 & 146 \\
\hline Fifth decile & 409 & 370 & 140 & 190 & 152 & 143 \\
\hline Sixth decile & 398 & 374 & 150 & 196 & 150 & 144 \\
\hline Seventh decile & 393 & 375 & 159 & 198 & 148 & 144 \\
\hline Eighth decile & 406 & 367 & 167 & 196 & 151 & 142 \\
\hline Ninth decile & 405 & 376 & 176 & 198 & 151 & 144 \\
\hline Highest $10 \%$ & 413 & 377 & 180 & 197 & 153 & 145 \\
\hline Highest 5\% & 415 & 383 & 175 & 197 & 154 & 146 \\
\hline Highest $2.5 \%$ & 415 & 389 & 168 & 196 & 154 & 147 \\
\hline All income units & 405 & 378 & 150 & 183 & 151 & 145 \\
\hline \multicolumn{7}{|c|}{ (c) Single parents } \\
\hline Lowest $\mathbf{5 \%}$ & 307 & 253 & 100 & 100 & 112 & 98 \\
\hline Lowest $10 \%$ & 282 & 245 & 100 & 100 & 106 & 96 \\
\hline Second decile & 275 & 268 & 100 & 100 & 104 & 102 \\
\hline Third decile & 299 & 239 & 100 & 100 & 110 & 95 \\
\hline Fourth decile & 245 & 253 & 100 & 100 & 96 & 98 \\
\hline Fifth decile & 253 & 251 & 100 & 100 & 98 & 98 \\
\hline Sixth decile & 247 & 225 & 100 & 100 & 97 & 91 \\
\hline Seventh decile & 270 & 241 & 100 & 100 & 103 & 95 \\
\hline Eighth decile & 292 & 225 & 100 & 100 & 108 & 91 \\
\hline
\end{tabular}


Measuring and Promoting Wellbeing: How Important is Economic Growth?

\begin{tabular}{|c|c|c|c|c|c|c|}
\hline \multicolumn{7}{|c|}{ Number per 100 income units } \\
\hline \multirow{2}{*}{ Private income } & \multicolumn{2}{|c|}{ Persons } & \multicolumn{2}{|c|}{\begin{tabular}{|l|} 
Employed persons \\
\end{tabular}} & \multicolumn{2}{|c|}{ Consumption units } \\
\hline & Australia & Sweden & Australia & Sweden & Australia & Sweden \\
\hline Ninth decile & 245 & 225 & 100 & 100 & 96 & 91 \\
\hline Highest $10 \%$ & 280 & 232 & 100 & 100 & 105 & 93 \\
\hline Highest $5 \%$ & 299 & 225 & 100 & 100 & 110 & 91 \\
\hline Highest $2.5 \%$ & 286 & 228 & 100 & 100 & 107 & 92 \\
\hline All income units & 269 & 241 & 100 & 100 & 102 & 95 \\
\hline \multicolumn{7}{|c|}{ (d) Single persons } \\
\hline Lowest $5 \%$ & 100 & 100 & 100 & 100 & 60 & 60 \\
\hline Lowest $10 \%$ & 100 & 100 & 100 & 100 & 60 & 60 \\
\hline Second decile & 100 & 100 & 100 & 100 & 60 & 60 \\
\hline Third decile & 100 & 100 & 100 & 100 & 60 & 60 \\
\hline Fourth decile & 100 & 100 & 100 & 100 & 60 & 60 \\
\hline Fifth decile & 100 & 100 & 100 & 100 & 60 & 60 \\
\hline Sixth decile & 100 & 100 & 100 & 100 & 60 & 60 \\
\hline Seventh decile & 100 & 100 & 100 & 100 & 60 & 60 \\
\hline Eighth decile & 100 & 100 & 100 & 100 & 60 & 60 \\
\hline Ninth decile & 100 & 100 & 100 & 100 & 60 & 60 \\
\hline Highest $10 \%$ & 100 & 100 & 100 & 100 & 60 & 60 \\
\hline Highest $5 \%$ & 100 & 100 & 100 & 100 & 60 & 60 \\
\hline Highest $2.5 \%$ & 100 & 100 & 100 & 100 & 60 & 60 \\
\hline All income units & 100 & 100 & 100 & 100 & 60 & 60 \\
\hline \multicolumn{7}{|c|}{ (e) All income units } \\
\hline Lowest 5\% & 214 & 154 & 116 & 104 & & \\
\hline Lowest $10 \%$ & 213 & 143 & 119 & 104 & & \\
\hline Second decile & 190 & 147 & 112 & 105 & & \\
\hline Third decile & 203 & 156 & 110 & 108 & & \\
\hline Fourth decile & 230 & 159 & 111 & 109 & & \\
\hline Fifth decile & 239 & 181 & 120 & 114 & & \\
\hline Sixth decile & 266 & 223 & 123 & 136 & & \\
\hline Seventh decile & 289 & 275 & 144 & 169 & & \\
\hline Eighth decile & 290 & 291 & 161 & 184 & & \\
\hline Ninth decile & 307 & 284 & 170 & 194 & & \\
\hline Highest $10 \%$ & 313 & 297 & 178 & 194 & & \\
\hline Highest $5 \%$ & 320 & 294 & 174 & 191 & & \\
\hline Highest $2.5 \%$ & 313 & 296 & 169 & 189 & & \\
\hline All income units & 254 & 215 & 135 & 141 & & \\
\hline
\end{tabular}


Table A10 Income units aged 20-64 with at least one employed adult: average real private income, by income unit type and private income quantile

\begin{tabular}{|c|c|c|c|c|}
\hline \multicolumn{5}{|c|}{ Average annual private income } \\
\hline Private income quantile & $\begin{array}{l}\text { Australia } \\
\text { (\$A'000) }\end{array}$ & $\begin{array}{l}\text { Sweden } \\
\text { (\$A'000) }\end{array}$ & $\begin{array}{l}\text { Australia } \\
\% \text { of total }\end{array}$ & $\begin{array}{l}\text { Sweden } \\
\% \text { of total }\end{array}$ \\
\hline \multicolumn{5}{|c|}{ (a) Married couples without children } \\
\hline Lowest $5 \%$ & 3.4 & 2.9 & 0.6 & 0.7 \\
\hline Lowest $10 \%$ & 7.2 & 5.4 & 2.4 & 2.4 \\
\hline Second decile & 14.9 & 11.5 & 5.1 & 5.1 \\
\hline Third decile & 18.4 & 14.8 & 6.4 & 6.6 \\
\hline Fourth decile & 22.4 & 17.6 & 7.6 & 7.9 \\
\hline Fifth decile & 26.2 & 20.3 & 9.0 & 9.1 \\
\hline Sixth decile & 29.4 & 23.1 & 10.0 & 10.3 \\
\hline Seventh decile & 32.7 & 25.5 & 11.2 & 11.4 \\
\hline Eighth decile & 36.7 & 27.9 & 12.6 & 12.5 \\
\hline Ninth decile & 42.6 & 31.8 & 14.5 & 14.2 \\
\hline Highest $10 \%$ & 61.2 & 45.8 & 21.2 & 20.5 \\
\hline Highest 5\% & 71.5 & 53.6 & 12.4 & 12.0 \\
\hline Highest $2.5 \%$ & 82.2 & 63.5 & 7.2 & 7.1 \\
\hline All income units & 29.2 & 22.4 & 100.0 & 100.0 \\
\hline Co-efficient of concentration & 0.278 & 0.268 & 0.278 & 0.268 \\
\hline \multicolumn{5}{|c|}{ (b) Married couples with children } \\
\hline Lowest $5 \%$ & 3.4 & 4.9 & 0.6 & 1.1 \\
\hline Lowest $10 \%$ & 7.1 & 8.1 & 2.6 & 3.5 \\
\hline Second decile & 14.7 & 13.9 & 5.4 & 6.0 \\
\hline Third decile & 17.3 & 16.7 & 6.4 & 7.2 \\
\hline Fourth decile & 20.0 & 19.0 & 7.4 & 8.2 \\
\hline Fifth decile & 23.0 & 21.0 & 8.6 & 9.1 \\
\hline Sixth decile & 26.1 & 23.2 & 9.7 & 10.1 \\
\hline Seventh decile & 29.4 & 25.3 & 10.9 & 10.9 \\
\hline Eighth decile & 33.3 & 27.8 & 12.3 & 12.0 \\
\hline Ninth decile & 39.5 & 31.8 & 14.6 & 13.8 \\
\hline Highest $10 \%$ & 59.6 & 44.2 & 22.1 & 19.2 \\
\hline Highest 5\% & 70.8 & 50.8 & 13.3 & 11.0 \\
\hline Highest $2.5 \%$ & 85.4 & 58.3 & 7.9 & 6.3 \\
\hline All income units & 27.0 & 23.1 & 100.0 & 100.0 \\
\hline Co-efficient of concentration & 0.281 & 0.229 & 0.281 & 0.229 \\
\hline \multicolumn{5}{|c|}{ (c) Single parents } \\
\hline Lowest $5 \%$ & 0.8 & 0.9 & 0.2 & 0.4 \\
\hline Lowest $10 \%$ & 1.4 & 1.9 & 0.9 & 1.8 \\
\hline Second decile & 3.5 & 5.0 & 2.2 & 4.9 \\
\hline Third decile & 6.8 & 7.0 & 4.6 & 6.9 \\
\hline Fourth decile & 10.5 & 8.4 & 6.8 & 8.2 \\
\hline Fifth decile & 13.6 & 9.7 & 9.4 & 9.5 \\
\hline Sixth decile & 16.6 & 11.0 & 10.7 & 10.7 \\
\hline Seventh decile & 18.9 & 11.9 & 12.2 & 11.6 \\
\hline Eighth decile & 21.2 & 13.0 & 14.4 & 12.7 \\
\hline Ninth decile & 24.5 & 14.7 & 16.2 & 14.3 \\
\hline Highest $10 \%$ & 33.7 & 20.0 & 22.6 & 19.4 \\
\hline
\end{tabular}




\begin{tabular}{|l|c|c|c|c|}
\hline \multicolumn{5}{|c|}{ Average annual private income } \\
\hline Private income quantile & $\begin{array}{c}\text { Australia } \\
\text { (\$A'000) }\end{array}$ & $\begin{array}{c}\text { Sweden } \\
\text { (\$A'000) }\end{array}$ & $\begin{array}{c}\text { Australia } \\
\text { \% of total }\end{array}$ & $\begin{array}{c}\text { Sweden } \\
\text { \% of total }\end{array}$ \\
\hline Highest 5\% & 37.0 & 22.8 & 12.5 & 11.1 \\
\hline Highest 2.5\% & 39.4 & 25.9 & 6.8 & 6.3 \\
\hline All income units & 15.1 & 10.3 & 100.0 & 100.0 \\
\hline Co-efficient of concentration & 0.360 & 0.265 & 0.360 & 0.265 \\
\hline \multicolumn{5}{|c|}{ (d) Single persons } \\
\hline Lowest 5\% & 1.2 & 1.7 & 0.3 & 0.8 \\
\hline Lowest 10\% & 3.2 & 2.8 & 1.9 & 2.6 \\
\hline Second decile & 9.9 & 5.2 & 6.0 & 4.8 \\
\hline Third decile & 12.7 & 7.1 & 7.6 & 6.5 \\
\hline Fourth decile & 14.0 & 8.9 & 8.5 & 8.1 \\
\hline Fifth decile & 15.1 & 10.5 & 9.2 & 9.6 \\
\hline Sixth decile & 16.4 & 11.7 & 9.9 & 10.7 \\
\hline Seventh decile & 18.0 & 12.6 & 10.9 & 11.4 \\
\hline Eighth decile & 19.9 & 13.9 & 12.0 & 12.7 \\
\hline Ninth decile & 23.0 & 15.5 & 13.8 & 14.0 \\
\hline Highest 10\% & 33.2 & 21.5 & 20.2 & 19.6 \\
\hline Highest 5\% & 38.8 & 25.0 & 11.8 & 11.4 \\
\hline Highest 2.5\% & 45.6 & 29.4 & 7.0 & 6.7 \\
\hline All income units & 16.5 & 11.0 & 100.0 & 100.0 \\
\hline Co-efficient of concentration & 0.249 & 0.259 & 0.249 & 0.259 \\
\hline & (e) All income units & & \\
\hline Lowest 5\% & 1.9 & 1.9 & 0.4 & 0.6 \\
\hline Lowest 10\% & 4.9 & 3.4 & 2.1 & 2.0 \\
\hline Second decile & 12.3 & 7.4 & 5.2 & 4.3 \\
\hline Third decile & 14.8 & 10.2 & 6.2 & 5.9 \\
\hline Fourth decile & 16.8 & 12.1 & 7.1 & 7.0 \\
\hline Fifth decile & 19.2 & 13.9 & 8.1 & 8.1 \\
\hline Sixth decile & 22.2 & 16.3 & 9.4 & 9.4 \\
\hline Seventh decile & 25.9 & 19.3 & 10.9 & 11.2 \\
\hline Eighth decile & 30.2 & 23.2 & 12.8 & 13.4 \\
\hline Ninth decile & 36.1 & 27.3 & 15.2 & 15.8 \\
\hline Highest 10\% & 54.5 & 39.2 & 23.0 & 22.9 \\
\hline Highest 5\% & 64.7 & 45.9 & 13.7 & 13.3 \\
\hline Highest 2.5\% & 76.7 & 17.2 & 100.0 & 100.0 \\
\hline All income units & 0.320 & 0.304 & 0.320 \\
\hline Co-efficient of concentration
\end{tabular}


Table A11 Income units in 20-64 age group with at least one employed adult: average real disposable income, by income unit type and private income quantile

\begin{tabular}{|c|c|c|c|c|}
\hline \multicolumn{5}{|c|}{ Average annual disposable income } \\
\hline Private income quantile & $\begin{array}{c}\text { Australia } \\
\$ A^{\prime} 000\end{array}$ & $\begin{array}{l}\text { Sweden } \\
\$ A^{\prime} 000\end{array}$ & $\begin{array}{c}\text { Australia } \\
\text { As \% of } \\
\text { total }\end{array}$ & $\begin{array}{c}\text { Sweden } \\
\text { As \% of } \\
\text { total }\end{array}$ \\
\hline \multicolumn{5}{|c|}{ (a) Married couples without children } \\
\hline Lowest $5 \%$ & 6.5 & 10.1 & 1.4 & 2.9 \\
\hline Lowest $10 \%$ & 8.6 & 11.6 & 3.8 & 6.7 \\
\hline Second decile & 13.2 & 12.8 & 5.9 & 7.5 \\
\hline Third decile & 15.5 & 13.7 & 7.0 & 8.0 \\
\hline Fourth decile & 18.2 & 14.4 & 8.0 & 8.4 \\
\hline Fifth decile & 20.9 & 15.5 & 9.3 & 9.0 \\
\hline Sixth decile & 23.0 & 16.8 & 10.2 & 9.8 \\
\hline Seventh decile & 25.4 & 18.1 & 11.3 & 10.5 \\
\hline Eighth decile & 28.0 & 19.7 & 12.4 & 11.4 \\
\hline Ninth decile & 31.5 & 21.5 & 13.8 & 12.5 \\
\hline Highest $10 \%$ & 40.9 & 27.7 & 18.3 & 16.2 \\
\hline Highest 5\% & 45.9 & 31.6 & 10.3 & 9.2 \\
\hline Highest $2.5 \%$ & 50.2 & 35.4 & 5.7 & 5.2 \\
\hline All income units & 22.5 & 17.2 & 100.0 & 100.0 \\
\hline Co-efficient of concentration & 0.224 & 0.145 & 0.224 & 0.145 \\
\hline \multicolumn{5}{|c|}{ (b) Married couples with children } \\
\hline Lowest $5 \%$ & 6.0 & 10.5 & 1.4 & 2.8 \\
\hline Lowest $10 \%$ & 8.6 & 12.3 & 4.0 & 6.5 \\
\hline Second decile & 13.9 & 14.6 & 6.4 & 7.8 \\
\hline Third decile & 15.5 & 15.9 & 7.1 & 8.4 \\
\hline Fourth decile & 17.5 & 17.0 & 8.0 & 9.0 \\
\hline Fifth decile & 19.4 & 17.4 & 9.0 & 9.2 \\
\hline Sixth decile & 21.4 & 18.9 & 9.9 & 10.0 \\
\hline Seventh decile & 23.5 & 19.9 & 10.8 & 10.6 \\
\hline Eighth decile & 26.2 & 21.0 & 12.1 & 11.1 \\
\hline Ninth decile & 30.2 & 22.9 & 13.9 & 12.3 \\
\hline Highest $10 \%$ & 40.8 & 28.4 & 18.8 & 15.1 \\
\hline Highest $5 \%$ & 46.2 & 31.2 & 10.8 & 8.3 \\
\hline Highest $2.5 \%$ & 52.7 & 34.7 & 6.1 & 4.6 \\
\hline All income units & 21.7 & 18.8 & 100.0 & 100.0 \\
\hline Co-efficient of concentration & 0.220 & 0.128 & 0.220 & 0.128 \\
\hline \multicolumn{5}{|c|}{ (c) Single parents } \\
\hline Lowest $5 \%$ & 7.4 & 10.3 & 2.5 & 4.3 \\
\hline Lowest $10 \%$ & 7.9 & 9.3 & 5.5 & 7.8 \\
\hline Second decile & 8.0 & 9.9 & 5.5 & 8.3 \\
\hline Third decile & 10.0 & 10.8 & 7.2 & 9.1 \\
\hline Fourth decile & 11.1 & 11.2 & 7.6 & 9.4 \\
\hline
\end{tabular}




\begin{tabular}{|c|c|c|c|c|}
\hline \multicolumn{5}{|c|}{ Average annual disposable income } \\
\hline Private income quantile & $\begin{array}{c}\text { Australia } \\
\$ A^{\prime} 000\end{array}$ & $\begin{array}{l}\text { Sweden } \\
\$ A^{\prime} 000\end{array}$ & $\begin{array}{c}\text { Australia } \\
\text { As \% of } \\
\text { total }\end{array}$ & $\begin{array}{c}\text { Sweden } \\
\text { As \% of } \\
\text { total }\end{array}$ \\
\hline Fifth decile & 12.8 & 11.6 & 9.5 & 9.7 \\
\hline Sixth decile & 14.3 & 12.2 & 9.8 & 10.2 \\
\hline Seventh decile & 16.4 & 12.4 & 11.2 & 10.4 \\
\hline Eighth decile & 17.7 & 12.4 & 13.0 & 10.4 \\
\hline Ninth decile & 19.1 & 13.8 & 13.4 & 11.3 \\
\hline Highest $10 \%$ & 24.2 & 16.0 & 17.3 & 13.4 \\
\hline Highest $5 \%$ & 25.8 & 16.9 & 9.3 & 7.1 \\
\hline Highest $2.5 \%$ & 26.5 & 18.3 & 4.9 & 3.8 \\
\hline All income units & 14.1 & 12.0 & 100.0 & 100.0 \\
\hline Co-efficient of concentration & 0.203 & 0.081 & 0.203 & 0.081 \\
\hline \multicolumn{5}{|c|}{ (d) Single persons } \\
\hline Lowest $5 \%$ & 3.1 & 5.5 & 1.2 & 3.3 \\
\hline Lowest $10 \%$ & 4.2 & 5.6 & 3.3 & 6.6 \\
\hline Second decile & 8.4 & 6.3 & 6.5 & 7.6 \\
\hline Third decile & 10.3 & 6.7 & 8.0 & 8.0 \\
\hline Fourth decile & 11.3 & 7.6 & 8.7 & 9.1 \\
\hline Fifth decile & 12.0 & 7.8 & 9.4 & 9.3 \\
\hline Sixth decile & 13.0 & 8.7 & 10.2 & 10.4 \\
\hline Seventh decile & 14.1 & 8.6 & 11.0 & 10.2 \\
\hline Eighth decile & 15.4 & 9.3 & 12.0 & 11.0 \\
\hline Ninth decile & 17.2 & 10.3 & 13.4 & 12.3 \\
\hline Highest $10 \%$ & 22.5 & 13.1 & 17.5 & 15.5 \\
\hline Highest $5 \%$ & 25.3 & 14.7 & 9.9 & 8.7 \\
\hline Highest $2.5 \%$ & 28.5 & 16.6 & 5.7 & 4.9 \\
\hline All income units & 12.8 & 8.4 & 100.0 & 100.0 \\
\hline Co-efficient of concentration & 0.204 & 0.132 & 0.204 & 0.132 \\
\hline \multicolumn{5}{|c|}{ (e) All income units } \\
\hline Lowest 5\% & 4.7 & 6.5 & 1.2 & 2.3 \\
\hline Lowest $10 \%$ & 6.4 & 6.8 & 3.4 & 4.9 \\
\hline Second decile & 10.8 & 8.5 & 5.7 & 6.2 \\
\hline Third decile & 12.6 & 9.3 & 6.7 & 6.7 \\
\hline Fourth decile & 14.2 & 10.1 & 7.6 & 7.3 \\
\hline Fifth decile & 15.9 & 11.4 & 8.5 & 8.2 \\
\hline Sixth decile & 18.0 & 13.3 & 9.6 & 9.6 \\
\hline Seventh decile & 20.7 & 15.6 & 11.0 & 11.3 \\
\hline Eighth decile & 23.6 & 17.7 & 12.6 & 12.8 \\
\hline Ninth decile & 27.6 & 19.9 & 14.7 & 14.4 \\
\hline Highest $10 \%$ & 37.7 & 25.5 & 20.2 & 18.6 \\
\hline Highest $5 \%$ & 42.7 & 28.3 & 11.4 & 10.3 \\
\hline Highest $2.5 \%$ & 48.1 & 31.6 & 6.4 & 5.7 \\
\hline All income units & 18.8 & 13.8 & 100.0 & 100.0 \\
\hline Co-efficient of concentration & 0.255 & 0.225 & 0.255 & 0.225 \\
\hline
\end{tabular}


Table A12 Income units in 20-64 age group with at least one employed adult: average real private and disposable income per consumption unit, by income unit type and private income quantile

\begin{tabular}{|c|c|c|c|c|}
\hline \multicolumn{5}{|c|}{ Average annual income per consumption unit (\$A'000) } \\
\hline \multirow{2}{*}{ Private income quantile } & \multicolumn{2}{|c|}{ Private income } & \multicolumn{2}{|c|}{ Disposable income } \\
\hline & Australia & Sweden & Australia & Sweden \\
\hline \multicolumn{5}{|c|}{ (a) Married couples without children } \\
\hline Lowest 5\% & 3.4 & 2.9 & 6.5 & 10.1 \\
\hline Lowest $10 \%$ & 7.2 & 5.4 & 8.6 & 11.6 \\
\hline Second decile & 14.9 & 11.5 & 13.2 & 12.8 \\
\hline Third decile & 18.4 & 14.8 & 15.5 & 13.7 \\
\hline Fourth decile & 22.4 & 17.6 & 18.2 & 14.4 \\
\hline Fifth decile & 26.2 & 20.3 & 20.9 & 15.5 \\
\hline Sixth decile & 29.4 & 23.1 & 23.0 & 16.8 \\
\hline Seventh decile & 32.7 & 25.5 & 25.4 & 18.1 \\
\hline Eighth decile & 36.7 & 27.9 & 28.0 & 19.7 \\
\hline Ninth decile & 42.6 & 31.8 & 31.5 & 21.5 \\
\hline Highest $10 \%$ & 61.2 & 45.8 & 40.9 & 27.7 \\
\hline Highest 5\% & 71.5 & 53.6 & 45.9 & 31.6 \\
\hline Highest $2.5 \%$ & 82.2 & 63.5 & 50.2 & 35.4 \\
\hline All income units & 29.2 & 22.4 & 22.5 & 17.2 \\
\hline \multicolumn{5}{|c|}{ (b) Married couples with children } \\
\hline Lowest 5\% & 2.3 & 3.5 & 3.9 & 7.5 \\
\hline Lowest $10 \%$ & 4.7 & 5.5 & 5.7 & 8.4 \\
\hline Second decile & 9.5 & 9.4 & 9.0 & 9.9 \\
\hline Third decile & 11.4 & 11.4 & 10.2 & 10.8 \\
\hline Fourth decile & 13.4 & 13.0 & 11.7 & 11.6 \\
\hline Fifth decile & 15.1 & 14.7 & 12.8 & 12.2 \\
\hline Sixth decile & 17.4 & 16.2 & 14.3 & 13.2 \\
\hline Seventh decile & 19.8 & 17.6 & 15.9 & 13.8 \\
\hline Eighth decile & 22.0 & 19.6 & 17.3 & 14.8 \\
\hline Ninth decile & 26.1 & 22.0 & 19.9 & 15.9 \\
\hline Highest $10 \%$ & 38.9 & 30.6 & 26.6 & 19.7 \\
\hline Highest 5\% & 46.0 & 34.9 & 30.0 & 21.4 \\
\hline Highest $2.5 \%$ & 55.6 & 39.5 & 34.3 & 23.5 \\
\hline All income units & 17.9 & 16.0 & 14.4 & 13.0 \\
\hline \multicolumn{5}{|c|}{ (c) Single parents } \\
\hline Lowest 5\% & 0.8 & 0.9 & 6.6 & 10.5 \\
\hline Lowest $10 \%$ & 1.4 & 1.9 & 7.5 & 9.7 \\
\hline Second decile & 3.4 & 4.9 & 7.7 & 9.8 \\
\hline Third decile & 6.3 & 7.4 & 9.1 & 11.4 \\
\hline
\end{tabular}


Measuring and Promoting Wellbeing: How Important is Economic Growth?

\begin{tabular}{|l|c|c|c|c|}
\hline \multicolumn{4}{|c|}{ Average annual income per consumption unit (\$A'000) } \\
\hline \multirow{2}{*}{ Private income quantile } & \multicolumn{2}{|c|}{ Private income } & \multicolumn{2}{c|}{ Disposable income } \\
\cline { 2 - 5 } & Australia & Sweden & Australia & Sweden \\
\hline Fourth decile & 10.9 & 8.5 & 11.6 & 11.4 \\
\hline Fifth decile & 13.7 & 9.9 & 13.1 & 11.9 \\
\hline Sixth decile & 17.2 & 12.0 & 14.1 & 13.3 \\
\hline Seventh decile & 18.3 & 12.5 & 16.9 & 13.0 \\
\hline Eighth decile & 19.7 & 14.3 & 16.5 & 13.6 \\
\hline Ninth decile & 25.6 & 16.1 & 19.9 & 15.1 \\
\hline Highest 10\% & 32.0 & 21.5 & 23.1 & 17.2 \\
\hline Highest 5\% & 33.7 & 25.0 & 23.5 & 18.5 \\
\hline Highest 2.5\% & 36.9 & 28.2 & 24.9 & 19.9 \\
\hline All income units & 14.7 & 10.8 & 13.8 & 12.6 \\
\hline & (d) Single persons & & \\
\hline Lowest 5\% & 1.9 & 2.8 & 5.2 & 9.2 \\
\hline Lowest 10\% & 5.3 & 4.7 & 7.0 & 9.3 \\
\hline Second decile & 16.5 & 8.7 & 14.0 & 10.6 \\
\hline Third decile & 21.1 & 11.9 & 17.2 & 11.2 \\
\hline Fourth decile & 23.3 & 14.8 & 18.8 & 12.7 \\
\hline Fifth decile & 25.1 & 17.5 & 20.0 & 13.1 \\
\hline Sixth decile & 27.4 & 19.6 & 21.6 & 14.5 \\
\hline Seventh decile & 30.0 & 20.9 & 23.5 & 14.3 \\
\hline Eighth decile & 33.2 & 22.2 & 25.7 & 15.6 \\
\hline Ninth decile & 38.3 & 25.8 & 28.6 & 17.2 \\
\hline Highest 10\% & 55.4 & 35.8 & 37.5 & 21.8 \\
\hline Highest 5\% & 27.6 & 41.7 & 42.1 & 24.4 \\
\hline Highest 2.5\% & 48.8 & 47.5 & 27.6 \\
\hline All income units & 18.3 & 21.4 & 14.0 \\
\hline
\end{tabular}


Table A13 Income units in 20-64 age group with at least one employed adult: average disposable income as \% of average private income, by income unit type and private income quantile

\begin{tabular}{|c|c|c|c|c|c|c|}
\hline \multicolumn{7}{|c|}{ Disposable income as \% of private income } \\
\hline $\begin{array}{l}\text { Private income } \\
\text { quantile }\end{array}$ & & $\begin{array}{l}\text { Married } \\
\text { couples } \\
\text { without } \\
\text { children }\end{array}$ & $\begin{array}{l}\text { Married } \\
\text { couples } \\
\text { with } \\
\text { children }\end{array}$ & $\begin{array}{l}\text { Single } \\
\text { parents }\end{array}$ & $\begin{array}{l}\text { Single } \\
\text { persons }\end{array}$ & $\begin{array}{l}\text { All } \\
\text { units }\end{array}$ \\
\hline Lowest $\mathbf{5 \%}$ & $\begin{array}{l}\text { Australia } \\
\text { Sweden }\end{array}$ & $\begin{array}{l}189.7 \\
346.7\end{array}$ & $\begin{array}{l}174.6 \\
214.2\end{array}$ & $\begin{array}{c}925.0 \\
1,144.0\end{array}$ & $\begin{array}{l}268.7 \\
324.5\end{array}$ & $\begin{array}{l}247.0 \\
342.0\end{array}$ \\
\hline Lowest $10 \%$ & $\begin{array}{l}\text { Australia } \\
\text { Sweden }\end{array}$ & $\begin{array}{l}119.8 \\
214.4\end{array}$ & $\begin{array}{l}121.3 \\
152.2\end{array}$ & $\begin{array}{l}564.0 \\
489.0\end{array}$ & $\begin{array}{l}132.3 \\
197.0\end{array}$ & $\begin{array}{l}130.6 \\
200.4\end{array}$ \\
\hline Second decile & $\begin{array}{l}\text { Australia } \\
\text { Sweden }\end{array}$ & $\begin{array}{c}88.2 \\
111.5\end{array}$ & $\begin{array}{c}94.5 \\
105.1\end{array}$ & $\begin{array}{l}235.0 \\
198.0\end{array}$ & $\begin{array}{c}84.6 \\
121.2\end{array}$ & $\begin{array}{r}87.8 \\
114.9\end{array}$ \\
\hline Third decile & $\begin{array}{l}\text { Australia } \\
\text { Sweden }\end{array}$ & $\begin{array}{l}84.3 \\
92.4\end{array}$ & $\begin{array}{l}89.8 \\
95.0\end{array}$ & $\begin{array}{l}147.0 \\
154.2\end{array}$ & $\begin{array}{l}81.5 \\
94.4\end{array}$ & $\begin{array}{l}84.9 \\
91.2\end{array}$ \\
\hline Fourth decile & $\begin{array}{l}\text { Australia } \\
\text { Sweden }\end{array}$ & $\begin{array}{l}81.5 \\
81.6\end{array}$ & $\begin{array}{l}87.3 \\
89.2\end{array}$ & $\begin{array}{l}105.9 \\
133.4\end{array}$ & $\begin{array}{l}80.5 \\
85.9\end{array}$ & $\begin{array}{l}84.3 \\
83.5\end{array}$ \\
\hline Fifth decile & $\begin{array}{l}\text { Australia } \\
\text { Sweden }\end{array}$ & $\begin{array}{l}79.7 \\
76.2\end{array}$ & $\begin{array}{l}84.4 \\
82.9\end{array}$ & $\begin{array}{c}94.3 \\
119.5\end{array}$ & $\begin{array}{l}80.1 \\
74.5\end{array}$ & $\begin{array}{l}82.9 \\
81.8\end{array}$ \\
\hline Sixth decile & $\begin{array}{l}\text { Australia } \\
\text { Sweden }\end{array}$ & $\begin{array}{l}78.5 \\
72.9\end{array}$ & $\begin{array}{l}82.1 \\
81.5\end{array}$ & $\begin{array}{c}86.5 \\
110.7\end{array}$ & $\begin{array}{l}79.1 \\
74.1\end{array}$ & $\begin{array}{l}81.3 \\
81.6\end{array}$ \\
\hline Seventh decile & $\begin{array}{l}\text { Australia } \\
\text { Sweden }\end{array}$ & $\begin{array}{l}78.0 \\
71.0\end{array}$ & $\begin{array}{l}80.1 \\
78.6\end{array}$ & $\begin{array}{c}86.6 \\
104.4\end{array}$ & $\begin{array}{l}78.2 \\
68.1\end{array}$ & $\begin{array}{l}79.7 \\
80.8\end{array}$ \\
\hline Eighth decile & $\begin{array}{l}\text { Australia } \\
\text { Sweden }\end{array}$ & $\begin{array}{l}76.2 \\
70.4\end{array}$ & $\begin{array}{l}78.7 \\
75.3\end{array}$ & $\begin{array}{l}83.6 \\
95.2\end{array}$ & $\begin{array}{l}77.2 \\
66.9\end{array}$ & $\begin{array}{l}78.0 \\
76.4\end{array}$ \\
\hline Ninth decile & $\begin{array}{l}\text { Australia } \\
\text { Sweden }\end{array}$ & $\begin{array}{l}73.8 \\
67.6\end{array}$ & $\begin{array}{l}76.4 \\
72.2\end{array}$ & $\begin{array}{l}77.8 \\
93.7\end{array}$ & $\begin{array}{l}74.7 \\
66.6\end{array}$ & $\begin{array}{l}76.4 \\
72.8\end{array}$ \\
\hline Highest $10 \%$ & $\begin{array}{l}\text { Australia } \\
\text { Sweden }\end{array}$ & $\begin{array}{l}66.9 \\
60.5\end{array}$ & $\begin{array}{l}68.5 \\
64.3\end{array}$ & $\begin{array}{l}71.7 \\
80.2\end{array}$ & $\begin{array}{l}67.7 \\
60.8\end{array}$ & $\begin{array}{l}69.1 \\
65.1\end{array}$ \\
\hline Highest $5 \%$ & $\begin{array}{l}\text { Australia } \\
\text { Sweden }\end{array}$ & $\begin{array}{l}64.2 \\
59.0\end{array}$ & $\begin{array}{l}65.2 \\
61.4\end{array}$ & $\begin{array}{l}69.7 \\
74.2\end{array}$ & $\begin{array}{l}65.2 \\
58.5\end{array}$ & $\begin{array}{l}66.0 \\
61.7\end{array}$ \\
\hline Highest $2.5 \%$ & $\begin{array}{l}\text { Australia } \\
\text { Sweden }\end{array}$ & $\begin{array}{l}61.1 \\
55.8\end{array}$ & $\begin{array}{l}61.7 \\
59.5\end{array}$ & $\begin{array}{l}67.4 \\
70.8\end{array}$ & $\begin{array}{l}62.5 \\
56.6\end{array}$ & $\begin{array}{l}62.8 \\
59.3\end{array}$ \\
\hline All income units & $\begin{array}{l}\text { Australia } \\
\text { Sweden }\end{array}$ & $\begin{array}{l}77.2 \\
76.8\end{array}$ & $\begin{array}{l}80.4 \\
81.5\end{array}$ & $\begin{array}{c}93.3 \\
116.7\end{array}$ & $\begin{array}{l}77.6 \\
76.6\end{array}$ & $\begin{array}{l}79.3 \\
80.1\end{array}$ \\
\hline
\end{tabular}


Measuring and Promoting Wellbeing: How Important is Economic Growth?

Table A14 Income units in 20-64 age group with at least one employed adult: average real private and disposable income per consumption unit (CU) by income unit type and private income quantile, Sweden as \% of Australia

\begin{tabular}{|c|c|c|c|c|}
\hline \multicolumn{5}{|c|}{ Sweden as \% of Australia } \\
\hline Private income quantile & $\begin{array}{l}\text { Married } \\
\text { couples } \\
\text { without } \\
\text { children }\end{array}$ & $\begin{array}{c}\text { Married } \\
\text { couples } \\
\text { with children }\end{array}$ & $\begin{array}{c}\text { Single } \\
\text { parents }\end{array}$ & $\begin{array}{c}\text { Single } \\
\text { persons }\end{array}$ \\
\hline \multicolumn{5}{|c|}{ (a) Average real private income per CU } \\
\hline Lowest $5 \%$ & 85.3 & 152.2 & 112.5 & 147.4 \\
\hline Lowest $10 \%$ & 75.0 & 117.0 & 135.7 & 88.7 \\
\hline Second decile & 77.1 & 99.2 & 144.1 & 52.9 \\
\hline Third decile & 80.6 & 99.9 & 117.5 & 56.4 \\
\hline Fourth decile & 78.7 & 97.0 & 78.0 & 63.5 \\
\hline Fifth decile & 77.7 & 97.4 & 72.3 & 69.7 \\
\hline Sixth decile & 78.5 & 92.8 & 69.9 & 71.5 \\
\hline Seventh decile & 78.3 & 88.7 & 68.3 & 69.8 \\
\hline Eighth decile & 76.2 & 89.2 & 72.4 & 66.9 \\
\hline Ninth decile & 74.5 & 84.6 & 62.9 & 67.2 \\
\hline Highest $10 \%$ & 74.8 & 78.7 & 67.2 & 64.6 \\
\hline Highest $5 \%$ & 74.9 & 75.8 & 74.1 & 64.5 \\
\hline Highest $2.5 \%$ & 77.3 & 71.1 & 76.2 & 64.2 \\
\hline All income units & 76.7 & 89.4 & 73.0 & 66.4 \\
\hline \multicolumn{5}{|c|}{ (b) Average real disposable income per CU } \\
\hline Lowest 5\% & 155.0 & 192.3 & 159.1 & 178.0 \\
\hline Lowest $10 \%$ & 134.9 & 147.4 & 129.3 & 132.7 \\
\hline Second decile & 97.4 & 110.4 & 127.3 & 75.8 \\
\hline Third decile & 88.4 & 105.7 & 125.4 & 65.3 \\
\hline Fourth decile & 78.8 & 99.1 & 98.0 & 67.8 \\
\hline Fifth decile & 74.3 & 95.8 & 90.8 & 65.2 \\
\hline Sixth decile & 72.9 & 92.0 & 94.3 & 67.0 \\
\hline Seventh decile & 71.2 & 87.0 & 93.4 & 60.8 \\
\hline Eighth decile & 70.4 & 85.4 & 82.4 & 60.7 \\
\hline Ninth decile & 68.3 & 79.8 & 75.7 & 59.9 \\
\hline Highest $10 \%$ & 67.7 & 73.9 & 74.3 & 58.0 \\
\hline Highest 5\% & 68.9 & 71.1 & 78.9 & 58.0 \\
\hline Highest $2.5 \%$ & 70.6 & 68.6 & 80.1 & 58.2 \\
\hline All income units & 76.3 & 90.3 & 91.3 & 65.5 \\
\hline
\end{tabular}


Table A15 Married couple income units without children, one employed: average real private and disposable income by income quantiles

\begin{tabular}{|c|c|c|c|c|}
\hline \multicolumn{5}{|c|}{ Average annual income } \\
\hline \multirow{2}{*}{ Income quantile } & \multicolumn{2}{|c|}{$\$ A^{\prime} 000$} & \multicolumn{2}{|c|}{ As $\%$ of total } \\
\hline & Australia & Sweden & Australia & Sweden \\
\hline \multicolumn{5}{|c|}{ (a) Private income, units ranked by private income } \\
\hline Lowest $10 \%$ & 3.9 & 2.0 & 1.7 & 1.6 \\
\hline Second decile & 11.5 & 5.4 & 5.2 & 4.1 \\
\hline Third decile & 14.5 & 7.1 & 6.6 & 5.5 \\
\hline Fourth decile & 16.1 & 8.9 & 7.3 & 6.8 \\
\hline Fifth decile & 17.9 & 10.8 & 8.0 & 8.2 \\
\hline Sixth decile & 20.3 & 12.4 & 9.2 & 9.5 \\
\hline Seventh decile & 23.2 & 14.1 & 10.5 & 10.8 \\
\hline Eighth decile & 26.5 & 16.1 & 11.8 & 12.3 \\
\hline Ninth decile & 32.0 & 18.4 & 14.5 & 14.1 \\
\hline Highest $10 \%$ & 55.8 & 35.3 & 25.2 & 27.0 \\
\hline Highest $5 \%$ & 70.0 & 48.8 & 15.7 & 18.7 \\
\hline All income units & 22.2 & 13.1 & 100.0 & 100.0 \\
\hline Co-efficient of concentration & 0.314 & 0.347 & 0.314 & 0.347 \\
\hline \multicolumn{5}{|c|}{ (b) Disposable income, units ranked by disposable income } \\
\hline Lowest $10 \%$ & 7.9 & 6.9 & 4.4 & 4.7 \\
\hline Second decile & 11.3 & 10.1 & 6.4 & 6.9 \\
\hline Third decile & 13.0 & 11.4 & 7.2 & 7.7 \\
\hline Fourth decile & 13.9 & 12.5 & 7.9 & 8.5 \\
\hline Fifth decile & 15.4 & 13.5 & 8.8 & 9.2 \\
\hline Sixth decile & 16.9 & 14.3 & 9.4 & 9.7 \\
\hline Seventh decile & 18.6 & 15.3 & 10.6 & 10.4 \\
\hline Eighth decile & 20.5 & 16.4 & 11.6 & 11.2 \\
\hline Ninth decile & 23.8 & 17.9 & 13.4 & 12.2 \\
\hline Highest $10 \%$ & 35.6 & 28.3 & 20.3 & 19.3 \\
\hline Highest $5 \%$ & 42.5 & 36.3 & 12.0 & 12.4 \\
\hline All income units & 17.7 & 14.7 & 100.0 & 100.0 \\
\hline Co-efficient concentration & 0.222 & 0.194 & 0.222 & 0.194 \\
\hline
\end{tabular}


Measuring and Promoting Wellbeing: How Important is Economic Growth?

Table A16 Married couple income units without children, both employed: average real private and disposable income by income quantiles

\begin{tabular}{|l|c|c|c|c|}
\hline \multicolumn{5}{|c|}{ Average annual income } \\
\hline \multirow{2}{*}{ Income quantile } & \multicolumn{2}{c|}{ \$A'000 } & \multicolumn{2}{c|}{ As \% of total } \\
\cline { 2 - 5 } & Australia & Sweden & Australia & Sweden \\
\hline \multicolumn{4}{|c|}{ (a) Private income, units ranked by private income } \\
\hline Lowest 10\% & 8.7 & 9.6 & 2.6 & 3.8 \\
\hline Second decile & 19.7 & 16.3 & 6.0 & 6.4 \\
\hline Third decile & 25.3 & 19.1 & 7.7 & 7.6 \\
\hline Fourth decile & 28.1 & 21.5 & 8.4 & 8.5 \\
\hline Fifth decile & 30.8 & 23.7 & 9.4 & 9.4 \\
\hline Sixth decile & 33.3 & 25.5 & 10.0 & 10.1 \\
\hline Seventh decile & 36.3 & 27.4 & 10.9 & 10.8 \\
\hline Eighth decile & 40.2 & 29.4 & 12.1 & 11.6 \\
\hline Ninth decile & 45.6 & 33.6 & 13.6 & 13.3 \\
\hline Highest 10\% & 63.0 & 47.0 & 19.3 & 18.6 \\
\hline Highest 5\% & 72.0 & 54.4 & 10.9 & 10.7 \\
\hline All income units & 33.1 & 25.3 & 100.0 & 100.0 \\
\hline Co-efficient of concentration & 0.234 & 0.208 & 0.234 & 0.208 \\
\hline & \multicolumn{5}{|c|}{ (b) Disposable income, units ranked by disposable income } & \\
\hline Lowest 10\% & 8.9 & 9.9 & 3.5 & 5.4 \\
\hline Second decile & 16.6 & 14.0 & 6.5 & 7.7 \\
\hline Third decile & 20.6 & 15.2 & 8.1 & 8.3 \\
\hline Fourth decile & 22.5 & 16.4 & 8.8 & 9.0 \\
\hline Fifth decile & 24.5 & 17.3 & 9.7 & 9.4 \\
\hline Sixth decile & 26.1 & 18.3 & 10.3 & 10.0 \\
\hline Seventh decile & 28.1 & 19.3 & 11.1 & 10.5 \\
\hline Eighth decile & 30.3 & 20.9 & 11.9 & 11.4 \\
\hline Ninth decile & 33.7 & 22.8 & 13.3 & 12.5 \\
\hline Highest 10\% & 42.6 & 29.0 & 16.8 & 15.8 \\
\hline Highest 5\% & 46.8 & 32.4 & 9.2 & 8.9 \\
\hline All income units & 25.4 & 18.3 & 100.0 & 100.0 \\
\hline Co-efficient of concentration & 0.194 & 0.148 & 0.194 & 0.148 \\
\hline
\end{tabular}


Table A17 Married couple income units with children: average real private and disposable income by income quantile

\begin{tabular}{|c|c|c|c|c|}
\hline \multicolumn{5}{|c|}{ Average annual income } \\
\hline \multirow{2}{*}{ Income quantile } & \multicolumn{2}{|c|}{$\$ A^{\prime} 000$} & \multicolumn{2}{|c|}{$\%$ of total } \\
\hline & Australia & Sweden & Australia & Sweden \\
\hline \multicolumn{5}{|c|}{ (a) Private income, units ranked by private income } \\
\hline Lowest $10 \%$ & 0.9 & 6.7 & 0.3 & 2.9 \\
\hline Second decile & 10.8 & 13.6 & 3.9 & 5.9 \\
\hline Third decile & 15.7 & 16.5 & 5.6 & 7.2 \\
\hline Fourth decile & 18.5 & 18.8 & 6.6 & 8.3 \\
\hline Fifth decile & 21.6 & 20.8 & 7.7 & 9.1 \\
\hline Sixth decile & 24.9 & 23.1 & 8.9 & 10.1 \\
\hline Seventh decile & 28.3 & 25.2 & 10.1 & 11.0 \\
\hline Eighth decile & 32.4 & 27.7 & 11.6 & 12.1 \\
\hline Ninth decile & 38.4 & 31.6 & 13.6 & 13.9 \\
\hline Highest $10 \%$ & 58.3 & 44.2 & 31.7 & 19.4 \\
\hline Highest $5 \%$ & 69.5 & 50.8 & 13.9 & 11.1 \\
\hline All income units & 25.0 & 22.8 & 100.0 & 100.0 \\
\hline Co-efficient of concentration & 0.393 & 0.239 & 0.393 & 0.239 \\
\hline \multicolumn{5}{|c|}{ (b) Disposable income, units ranked by disposable income } \\
\hline Lowest $10 \%$ & 7.2 & 9.8 & 3.5 & 5.2 \\
\hline Second decile & 12.1 & 14.0 & 5.8 & 7.5 \\
\hline Third decile & 14.6 & 15.6 & 7.0 & 8.3 \\
\hline Fourth decile & 16.5 & 16.7 & 8.0 & 9.0 \\
\hline Fifth decile & 18.5 & 17.8 & 8.9 & 9.5 \\
\hline Sixth decile & 20.6 & 19.0 & 9.9 & 10.1 \\
\hline Seventh decile & 22.9 & 20.1 & 11.0 & 10.7 \\
\hline Eighth decile & 25.6 & 21.4 & 12.3 & 11.4 \\
\hline Ninth decile & 29.5 & 23.5 & 14.1 & 12.6 \\
\hline Highest $10 \%$ & 40.3 & 29.5 & 19.5 & 15.8 \\
\hline Highest $5 \%$ & 45.8 & 32.9 & 11.0 & 8.8 \\
\hline All income units & 20.8 & 18.7 & 100.0 & 100.0 \\
\hline Co-efficient of concentration & 0.239 & 0.151 & 0.239 & 0.151 \\
\hline
\end{tabular}


Measuring and Promoting Wellbeing: How Important is Economic Growth?

Table A18 Married couple income units with children, one employed: average real private and disposable incomes by income quantiles

\begin{tabular}{|c|c|c|c|c|}
\hline \multicolumn{5}{|c|}{ Average annual income } \\
\hline \multirow{2}{*}{ Income quantile } & \multicolumn{2}{|c|}{$\$ A^{\prime} 000$} & \multicolumn{2}{|c|}{$\%$ of total } \\
\hline & Australia & Sweden & Australia & Sweden \\
\hline \multicolumn{5}{|c|}{ (a) Private income, units ranked by private income } \\
\hline Lowest $10 \%$ & 6.6 & 1.9 & 2.9 & 1.3 \\
\hline Second decile & 13.9 & 8.3 & 6.2 & 5.7 \\
\hline Third decile & 15.9 & 11.4 & 7.0 & 7.8 \\
\hline Fourth decile & 17.5 & 12.7 & 7.7 & 8.7 \\
\hline Fifth decile & 19.4 & 13.6 & 8.6 & 9.4 \\
\hline Sixth decile & 21.7 & 14.6 & 9.6 & 10.1 \\
\hline Seventh decile & 24.2 & 15.8 & 10.6 & 10.8 \\
\hline Eighth decile & 27.1 & 17.3 & 12.0 & 11.9 \\
\hline Ninth decile & 31.7 & 19.8 & 14.0 & 13.6 \\
\hline Highest $10 \%$ & 48.2 & 30.0 & 21.4 & 20.6 \\
\hline Highest $5 \%$ & 58.6 & 36.7 & 13.0 & 12.6 \\
\hline All Income Units & 22.6 & 14.5 & 100.0 & 100.0 \\
\hline Co-efficient of concentration & 0.256 & 0.257 & 0.256 & 0.257 \\
\hline \multicolumn{5}{|c|}{ (b) Disposable income, units ranked by disposable income } \\
\hline Lowest $10 \%$ & 8.5 & 6.3 & 4.6 & 4.2 \\
\hline Second decile & 13.1 & 11.3 & 7.0 & 7.6 \\
\hline Third decile & 14.6 & 12.4 & 7.8 & 8.4 \\
\hline Fourth decile & 15.7 & 13.4 & 8.4 & 9.0 \\
\hline Fifth decile & 17.1 & 14.2 & 9.1 & 9.5 \\
\hline Sixth decile & 18.5 & 15.1 & 9.9 & 10.1 \\
\hline Seventh decile & 19.9 & 16.0 & 10.7 & 10.8 \\
\hline Eighth decile & 21.7 & 17.1 & 11.4 & 11.5 \\
\hline Ninth decile & 24.4 & 19.0 & 13.3 & 12.8 \\
\hline Highest $10 \%$ & 33.1 & 23.8 & 17.8 & 16.0 \\
\hline Highest 5\% & 38.4 & 26.7 & 10.3 & 9.0 \\
\hline All income units & 18.7 & 14.9 & 100.0 & 100.0 \\
\hline Co-efficient of concentration & 0.188 & 0.165 & 0.188 & 0.165 \\
\hline
\end{tabular}


Table A19 Married couple income units with children, both employed: average real private and disposable incomes by income quantiles

\begin{tabular}{|c|c|c|c|c|}
\hline \multicolumn{5}{|c|}{ Average annual income } \\
\hline \multirow{2}{*}{ Income quantile } & \multicolumn{2}{|c|}{ In \$ $A^{\prime} 000$} & \multicolumn{2}{|c|}{$\%$ of total } \\
\hline & Australia & Sweden & Australia & Sweden \\
\hline \multicolumn{5}{|c|}{ (a) Private income, units ranked by private income } \\
\hline Lowest $10 \%$ & 7.7 & 10.6 & 2.4 & 4.3 \\
\hline Second decile & 16.6 & 16.4 & 5.3 & 6.6 \\
\hline Third decile & 21.2 & 18.9 & 6.7 & 7.6 \\
\hline Fourth decile & 25.0 & 20.7 & 8.0 & 8.3 \\
\hline Fifth decile & 28.1 & 22.7 & 9.0 & 9.2 \\
\hline Sixth decile & 31.1 & 24.5 & 9.9 & 9.9 \\
\hline Seventh decile & 34.3 & 26.4 & 11.0 & 10.7 \\
\hline Eighth decile & 38.6 & 28.9 & 12.2 & 11.7 \\
\hline Ninth decile & 45.3 & 33.0 & 14.5 & 13.3 \\
\hline Highest $10 \%$ & 66.0 & 45.4 & 21.0 & 18.3 \\
\hline Highest $5 \%$ & 79.1 & 51.9 & 12.6 & 10.5 \\
\hline All income units & 31.4 & 24.8 & 100.0 & 100.0 \\
\hline Co-efficient of concentration & 0.269 & 0.202 & 0.269 & 0.202 \\
\hline \multicolumn{5}{|c|}{ (b) Disposable income, units ranked by disposable income } \\
\hline Lowest $10 \%$ & 8.4 & 11.7 & 3.4 & 6.0 \\
\hline Second decile & 15.2 & 15.3 & 6.1 & 7.8 \\
\hline Third decile & 18.4 & 16.6 & 7.5 & 8.4 \\
\hline Fourth decile & 21.1 & 17.5 & 8.5 & 8.9 \\
\hline Fifth decile & 23.1 & 18.6 & 9.3 & 9.5 \\
\hline Sixth decile & 25.1 & 19.6 & 10.2 & 10.0 \\
\hline Seventh decile & 27.2 & 20.7 & 11.0 & 10.5 \\
\hline Eighth decile & 30.0 & 22.0 & 12.1 & 11.2 \\
\hline Ninth decile & 34.1 & 24.1 & 13.8 & 12.3 \\
\hline Highest $10 \%$ & 44.8 & 30.1 & 18.1 & 15.4 \\
\hline Highest $5 \%$ & 51.3 & 33.5 & 10.4 & 8.5 \\
\hline All income units & 24.7 & 19.6 & 100.0 & 100.0 \\
\hline Co-efficient of concentration & 0.218 & 0.135 & 0.218 & 0.135 \\
\hline
\end{tabular}


Table A20 Married couple income units with one child: average real private and disposable income by income quantiles

\begin{tabular}{|l|c|c|c|c|}
\hline \multirow{4}{*}{ Income quantile } & \multicolumn{2}{|c|}{ \$ A'000 } & \multicolumn{2}{c|}{$\%$ of total } \\
\hline \multicolumn{4}{|c|}{ (a) Private income, units ranked by private income } \\
\cline { 2 - 5 } & Australia & Sweden & Australia & Sweden \\
\hline Lowest 10\% & 0.3 & 5.7 & 0.1 & 2.5 \\
\hline Second decile & 7.8 & 13.2 & 3.3 & 5.8 \\
\hline Third decile & 14.5 & 16.4 & 6.0 & 7.3 \\
\hline Fourth decile & 17.9 & 18.9 & 7.6 & 8.4 \\
\hline Fifth decile & 20.8 & 20.8 & 8.7 & 9.2 \\
\hline Sixth decile & 24.5 & 22.9 & 10.2 & 10.1 \\
\hline Seventh decile & 28.1 & 25.3 & 11.8 & 11.2 \\
\hline Eighth decile & 32.0 & 27.8 & 13.4 & 12.3 \\
\hline Ninth decile & 37.5 & 31.6 & 15.7 & 14.0 \\
\hline Highest 10\% & 55.5 & 43.3 & 23.2 & 19.2 \\
\hline Highest 5\% & 65.6 & 48.9 & 13.7 & 10.8 \\
\hline All income units & 23.9 & 22.6 & 100.0 & 100.0 \\
\hline Co-efficient of concentration & 0.348 & 0.242 & 0.348 & 0.242 \\
\hline & \multicolumn{5}{|c|}{ (b) Disposable income, units ranked by disposable income } \\
\hline Lowest 10\% & 6.3 & 9.4 & 3.1 & 5.2 \\
\hline Second decile & 10.3 & 13.6 & 5.2 & 7.5 \\
\hline Third decile & 13.5 & 14.9 & 6.8 & 8.3 \\
\hline Fourth decile & 15.7 & 16.1 & 7.9 & 8.9 \\
\hline Fifth decile & 17.7 & 17.2 & 9.0 & 9.5 \\
\hline Sixth decile & 20.0 & 18.3 & 10.1 & 10.1 \\
\hline Seventh decile & 22.4 & 19.6 & 11.3 & 10.8 \\
\hline Eighth decile & 25.1 & 20.9 & 12.5 & 11.5 \\
\hline Ninth decile & 28.7 & 22.9 & 14.6 & 12.6 \\
\hline Highest 10\% & 38.6 & 28.1 & 19.5 & 15.5 \\
\hline Highest 5\% & 43.4 & 30.9 & 10.9 & 8.5 \\
\hline All income units & 18.1 & 100.0 & 100.0 \\
\hline Co-efficient of concentration & 0.152 & 0.253 & 0.152 \\
\hline
\end{tabular}


Table A21 Married couple income units with two children: average real private and disposable income by income quantiles

\begin{tabular}{|c|c|c|c|c|}
\hline \multicolumn{5}{|c|}{ Average annual income } \\
\hline \multirow{2}{*}{ Income quantile } & \multicolumn{2}{|c|}{$\$ A^{\prime} 000$} & \multicolumn{2}{|c|}{$\%$ of total } \\
\hline & Australia & Sweden & Australia & Sweden \\
\hline \multicolumn{5}{|c|}{ (a) Private income, units ranked by private income } \\
\hline Lowest $10 \%$ & 2.9 & 7.7 & 1.1 & 3.3 \\
\hline Second decile & 13.3 & 14.2 & 5.2 & 6.1 \\
\hline Third decile & 16.3 & 17.2 & 6.4 & 7.4 \\
\hline Fourth decile & 19.2 & 19.6 & 7.5 & 8.4 \\
\hline Fifth decile & 22.2 & 21.6 & 8.7 & 9.3 \\
\hline Sixth decile & 25.2 & 23.7 & 9.9 & 10.2 \\
\hline Seventh decile & 28.1 & 25.4 & 10.9 & 10.9 \\
\hline Eighth decile & 31.9 & 28.0 & 12.5 & 12.0 \\
\hline Ninth decile & 38.2 & 31.7 & 15.0 & 13.7 \\
\hline Highest $10 \%$ & 58.4 & 43.4 & 22.8 & 18.7 \\
\hline Highest $5 \%$ & 69.2 & 49.6 & 13.5 & 10.7 \\
\hline All income units & 25.6 & 23.2 & 100.0 & 100.0 \\
\hline Co-efficient of concentration & 0.306 & 0.223 & 0.306 & 0.223 \\
\hline \multicolumn{5}{|c|}{ (b) Disposable income, units ranked by disposable income } \\
\hline Lowest $10 \%$ & 7.8 & 9.5 & 3.7 & 5.0 \\
\hline Second decile & 13.0 & 14.2 & 6.2 & 7.6 \\
\hline Third decile & 14.8 & 15.8 & 7.1 & 8.4 \\
\hline Fourth decile & 16.8 & 16.9 & 8.0 & 9.0 \\
\hline Fifth decile & 18.8 & 18.0 & 9.0 & 9.6 \\
\hline Sixth decile & 20.6 & 19.1 & 9.9 & 10.1 \\
\hline Seventh decile & 22.7 & 20.0 & 10.8 & 10.7 \\
\hline Eighth decile & 25.2 & 21.4 & 12.1 & 11.4 \\
\hline Ninth decile & 29.1 & 23.5 & 14.0 & 12.5 \\
\hline Highest $10 \%$ & 40.1 & 29.4 & 19.2 & 15.7 \\
\hline Highest $5 \%$ & 45.6 & 32.9 & 10.9 & 8.8 \\
\hline All income units & 20.9 & 18.8 & 100.0 & 100.0 \\
\hline Co-efficient of concentration & 0.228 & 0.150 & 0.228 & 0.150 \\
\hline
\end{tabular}


Measuring and Promoting Wellbeing: How Important is Economic Growth?

Table A22 Married couple income units with three children: average real private and disposable income by income quantiles

\begin{tabular}{|c|c|c|c|c|}
\hline \multicolumn{5}{|c|}{ Average annual income } \\
\hline \multirow{2}{*}{ Income quantile } & \multicolumn{2}{|c|}{$\$ A^{\prime} 000$} & \multicolumn{2}{|c|}{$\%$ of total } \\
\hline & Australia & Sweden & Australia & Sweden \\
\hline \multicolumn{5}{|c|}{ (a) Private income, units ranked by private income } \\
\hline Lowest $10 \%$ & 1.4 & 8.4 & 0.5 & 3.6 \\
\hline Second decile & 12.5 & 13.5 & 4.8 & 5.8 \\
\hline Third decile & 16.6 & 16.4 & 6.3 & 7.1 \\
\hline Fourth decile & 19.3 & 17.9 & 7.2 & 7.7 \\
\hline Fifth decile & 22.6 & 19.5 & 8.5 & 8.4 \\
\hline Sixth decile & 25.8 & 22.3 & 9.7 & 9.6 \\
\hline Seventh decile & 30.2 & 24.8 & 11.6 & 10.7 \\
\hline Eighth decile & 34.3 & 27.4 & 12.8 & 11.8 \\
\hline Ninth decile & 40.3 & 32.2 & 14.9 & 13.9 \\
\hline Highest $10 \%$ & 61.0 & 49.6 & 23.7 & 21.4 \\
\hline Highest 5\% & 73.2 & 60.5 & 13.8 & 13.0 \\
\hline All income units & 26.4 & 23.2 & 100.0 & 100.0 \\
\hline Co-efficient of concentration & 0.327 & 0.251 & 0.327 & 0.251 \\
\hline \multicolumn{5}{|c|}{ (b) Disposable income, units ranked by disposable income } \\
\hline Lowest $10 \%$ & 7.7 & 11.7 & 3.5 & 5.8 \\
\hline Second decile & 13.1 & 15.1 & 6.0 & 7.5 \\
\hline Third decile & 15.6 & 16.5 & 7.0 & 8.1 \\
\hline Fourth decile & 17.7 & 17.7 & 7.9 & 8.8 \\
\hline Fifth decile & 19.6 & 18.9 & 9.0 & 9.4 \\
\hline Sixth decile & 21.7 & 20.0 & 9.8 & 9.9 \\
\hline Seventh decile & 24.5 & 21.2 & 11.1 & 10.5 \\
\hline Eighth decile & 27.3 & 22.8 & 12.3 & 11.3 \\
\hline Ninth decile & 31.1 & 25.0 & 14.0 & 12.4 \\
\hline Highest $10 \%$ & 42.4 & 33.0 & 19.4 & 16.3 \\
\hline Highest $5 \%$ & 48.3 & 38.4 & 10.9 & 9.5 \\
\hline All income units & 22.1 & 20.2 & 100.0 & 100.0 \\
\hline Co-efficient of concentration & 0.237 & 0.150 & 0.237 & 0.150 \\
\hline
\end{tabular}


Table A23 All single parent income units: average real private and disposable income by income quantiles

\begin{tabular}{|c|c|c|c|c|}
\hline \multicolumn{5}{|c|}{ Average annual income } \\
\hline \multirow{2}{*}{ Income quantile } & \multicolumn{2}{|c|}{$\$ A^{\prime} 000$} & \multicolumn{2}{|c|}{$\%$ of total } \\
\hline & Australia & Sweden & Australia & Sweden \\
\hline \multicolumn{5}{|c|}{ (a) Private income, units ranked by private income } \\
\hline Lowest $10 \%$ & 0.0 & 0.0 & 0.0 & 0.0 \\
\hline Second decile & 0.0 & 1.7 & 0.0 & 1.9 \\
\hline Third decile & 0.0 & 4.2 & 0.0 & 4.8 \\
\hline Fourth decile & 0.1 & 6.7 & 0.2 & 7.6 \\
\hline Fifth decile & 1.2 & 8.4 & 1.8 & 9.5 \\
\hline Sixth decile & 2.3 & 10.0 & 3.4 & 11.3 \\
\hline Seventh decile & 5.3 & 11.3 & 8.4 & 12.8 \\
\hline Eighth decile & 10.4 & 12.5 & 16.5 & 14.2 \\
\hline Ninth decile & 17.5 & 14.1 & 27.0 & 16.0 \\
\hline Highest $10 \%$ & 27.1 & 19.3 & 42.7 & 21.9 \\
\hline Highest $5 \%$ & 31.3 & 22.1 & 24.4 & 12.5 \\
\hline All income units & 6.4 & 8.8 & 100.0 & 100.0 \\
\hline Co-efficient of concentration & 0.682 & 0.360 & 0.682 & 0.360 \\
\hline \multicolumn{5}{|c|}{ (b) Disposable income, units ranked by disposable income } \\
\hline Lowest $10 \%$ & 4.0 & 5.8 & 4.0 & 5.0 \\
\hline Second decile & 6.2 & 8.5 & 6.2 & 7.3 \\
\hline Third decile & 6.6 & 9.8 & 6.6 & 8.4 \\
\hline Fourth decile & 7.2 & 10.5 & 7.2 & 9.1 \\
\hline Fifth decile & 7.9 & 11.0 & 8.1 & 9.5 \\
\hline Sixth decile & 8.8 & 11.7 & 8.8 & 10.1 \\
\hline Seventh decile & 10.0 & 12.5 & 10.1 & 10.7 \\
\hline Eighth decile & 12.1 & 13.4 & 12.1 & 11.6 \\
\hline Ninth decile & 15.6 & 14.8 & 15.7 & 12.8 \\
\hline Highest $10 \%$ & 21.0 & 17.9 & 21.2 & 15.5 \\
\hline Highest $5 \%$ & 23.2 & 19.7 & 11.7 & 8.5 \\
\hline All income units & 9.9 & 11.6 & 100.0 & 100.0 \\
\hline Co-efficient of concentration & 0.257 & 0.154 & 0.257 & 0.154 \\
\hline
\end{tabular}


Measuring and Promoting Wellbeing: How Important is Economic Growth?

Table A24 Single parent income units, parent employed: average real private and disposable income by income quantiles

\begin{tabular}{|c|c|c|c|c|}
\hline \multicolumn{5}{|c|}{ Average annual income } \\
\hline \multirow{2}{*}{ Income quantile } & \multicolumn{2}{|c|}{$\$ A^{\prime} 000$} & \multicolumn{2}{|c|}{$\%$ of total } \\
\hline & Australia & Sweden & Australia & Sweden \\
\hline \multicolumn{5}{|c|}{ (a) Private income, units ranked by private income } \\
\hline Lowest $10 \%$ & 1.4 & 1.8 & 0.9 & 1.7 \\
\hline Second decile & 3.4 & 5.0 & 2.2 & 4.9 \\
\hline Third decile & 6.8 & 7.0 & 4.6 & 6.8 \\
\hline Fourth decile & 10.4 & 8.3 & 6.7 & 8.2 \\
\hline Fifth decile & 13.5 & 9.7 & 9.4 & 9.5 \\
\hline Sixth decile & 16.5 & 11.0 & 10.7 & 10.7 \\
\hline Seventh decile & 18.8 & 12.0 & 12.1 & 11.6 \\
\hline Eighth decile & 21.1 & 13.0 & 14.5 & 12.7 \\
\hline Ninth decile & 24.3 & 14.7 & 16.2 & 14.3 \\
\hline Highest $10 \%$ & 33.5 & 19.9 & 22.7 & 19.5 \\
\hline Highest 5\% & 37.0 & 22.8 & 12.3 & 11.1 \\
\hline All income units & 15.1 & 10.2 & 100.0 & 100.0 \\
\hline Co-efficient of concentration & 0.360 & 0.268 & 0.360 & 0.268 \\
\hline \multicolumn{5}{|c|}{ (b) Disposable income, units ranked by disposable income } \\
\hline Lowest $10 \%$ & 6.1 & 6.8 & 4.3 & 5.7 \\
\hline Second decile & 8.8 & 9.2 & 6.2 & 7.7 \\
\hline Third decile & 10.1 & 10.2 & 6.8 & 8.5 \\
\hline Fourth decile & 11.2 & 10.8 & 8.1 & 9.0 \\
\hline Fifth decile & 12.8 & 11.2 & 9.3 & 9.4 \\
\hline Sixth decile & 14.6 & 11.9 & 10.6 & 10.0 \\
\hline Seventh decile & 16.1 & 12.6 & 10.8 & 10.6 \\
\hline Eighth decile & 17.9 & 13.6 & 13.2 & 11.4 \\
\hline Ninth decile & 19.2 & 14.9 & 13.2 & 12.5 \\
\hline Highest $10 \%$ & 24.0 & 18.1 & 17.7 & 15.2 \\
\hline Highest $5 \%$ & 25.8 & 20.0 & 9.1 & 8.4 \\
\hline All income units & 14.1 & 11.9 & 100.0 & 100.0 \\
\hline Co-efficient of concentration & 0.212 & 0.139 & 0.212 & 0.139 \\
\hline
\end{tabular}


Table A25 Single person income units, person employed: average real private and disposable income by income quantiles

\begin{tabular}{|c|c|c|c|c|}
\hline \multicolumn{5}{|c|}{ Average annual income } \\
\hline \multirow{2}{*}{ Income quantile } & \multicolumn{2}{|c|}{$\$ A^{\prime} 000$} & \multicolumn{2}{|c|}{$\%$ of total } \\
\hline & Australia & Sweden & Australia & Sweden \\
\hline \multicolumn{5}{|c|}{ (a) Private income, units ranked by private income } \\
\hline Lowest $10 \%$ & 2.5 & 2.7 & 1.6 & 2.6 \\
\hline Second decile & 8.0 & 4.7 & 5.3 & 4.4 \\
\hline Third decile & 10.6 & 6.3 & 7.0 & 6.1 \\
\hline Fourth decile & 12.6 & 8.0 & 8.2 & 7.6 \\
\hline Fifth decile & 14.0 & 9.7 & 9.2 & 9.3 \\
\hline Sixth decile & 15.3 & 11.3 & 10.1 & 10.8 \\
\hline Seventh decile & 16.9 & 12.3 & 11.1 & 11.7 \\
\hline Eighth decile & 18.8 & 13.6 & 12.3 & 13.0 \\
\hline Ninth decile & 21.9 & 15.2 & 14.3 & 14.5 \\
\hline Highest $10 \%$ & 31.9 & 21.0 & 20.9 & 20.0 \\
\hline Highest $5 \%$ & 37.4 & 24.4 & 12.3 & 11.7 \\
\hline All income units & 15.3 & 10.5 & 100.0 & 100.0 \\
\hline Co-efficient of concentration & 0.273 & 0.276 & 0.273 & 0.276 \\
\hline \multicolumn{5}{|c|}{ (b) Disposable income, units ranked by disposable income } \\
\hline Lowest $10 \%$ & 3.8 & 3.5 & 3.1 & 4.4 \\
\hline Second decile & 7.2 & 5.3 & 6.0 & 6.6 \\
\hline Third decile & 8.9 & 6.6 & 7.4 & 8.1 \\
\hline Fourth decile & 10.3 & 7.3 & 8.5 & 9.0 \\
\hline Fifth decile & 11.3 & 7.8 & 9.4 & 9.7 \\
\hline Sixth decile & 12.2 & 8.3 & 10.2 & 10.2 \\
\hline Seventh decile & 13.3 & 8.8 & 11.1 & 10.8 \\
\hline Eighth decile & 14.7 & 9.5 & 12.2 & 11.7 \\
\hline Ninth decile & 16.6 & 10.4 & 13.8 & 12.9 \\
\hline Highest $10 \%$ & 21.8 & 13.4 & 18.3 & 16.6 \\
\hline Highest $5 \%$ & 24.6 & 15.2 & 10.3 & 9.4 \\
\hline All income units & 12.0 & 8.1 & 100.0 & 100.0 \\
\hline Co-efficient of concentration & 0.223 & 0.178 & 0.223 & 0.178 \\
\hline
\end{tabular}


Measuring and Promoting Wellbeing: How Important is Economic Growth?

Table A26 Income units in 18-19 years of age group: average real private and disposable income by income quantiles

\begin{tabular}{|c|c|c|c|c|}
\hline \multicolumn{5}{|c|}{ Average annual income } \\
\hline \multirow[t]{2}{*}{ Income quantile } & \multicolumn{2}{|c|}{$\$ A^{\prime} 000$} & \multicolumn{2}{|c|}{$\%$ of total } \\
\hline & Australia & Sweden & Australia & Sweden \\
\hline \multicolumn{5}{|c|}{ (a) Private income, units ranked by private income } \\
\hline Lowest $10 \%$ & 0.0 & 0.0 & 0.0 & 0.0 \\
\hline Second decile & 0.0 & 0.8 & 0.0 & 2.1 \\
\hline Third decile & 0.0 & 1.4 & 0.0 & 4.0 \\
\hline Fourth decile & 0.8 & 2.0 & 1.3 & 5.5 \\
\hline Fifth decile & 5.6 & 2.5 & 8.9 & 7.0 \\
\hline Sixth decile & 8.2 & 3.0 & 13.2 & 8.5 \\
\hline Seventh decile & 9.5 & 4.0 & 15.4 & 11.3 \\
\hline Eighth decile & 10.5 & 4.8 & 16.7 & 13.4 \\
\hline Ninth decile & 11.7 & 6.8 & 19.0 & 19.0 \\
\hline Highest $10 \%$ & 15.8 & 10.4 & 25.5 & 29.2 \\
\hline Highest $5 \%$ & 17.5 & 12.1 & 14.2 & 17.0 \\
\hline All income units & 6.2 & 3.6 & 100.0 & 100.0 \\
\hline Co-efficient of concentration & 0.493 & 0.446 & 0.493 & 0.446 \\
\hline \multicolumn{5}{|c|}{ (b) Disposable income, units ranked by disposable income } \\
\hline Lowest $10 \%$ & 0.0 & 0.4 & 0.0 & 1.2 \\
\hline Second decile & 0.5 & 1.2 & 0.8 & 3.6 \\
\hline Third decile & 3.1 & 1.7 & 5.1 & 5.2 \\
\hline Fourth decile & 4.2 & 2.2 & 6.9 & 6.8 \\
\hline Fifth decile & 6.0 & 2.7 & 10.1 & 8.1 \\
\hline Sixth decile & 7.2 & 3.2 & 12.1 & 9.9 \\
\hline Seventh decile & 8.1 & 3.7 & 13.3 & 11.2 \\
\hline Eighth decile & 8.8 & 4.4 & 14.7 & 13.4 \\
\hline Ninth decile & 9.7 & 5.7 & 16.0 & 17.5 \\
\hline Highest $10 \%$ & 12.5 & 7.6 & 21.0 & 23.1 \\
\hline Highest 5\% & 13.7 & 8.3 & 11.6 & 12.7 \\
\hline All income units & 6.0 & 3.3 & 100.0 & 100.0 \\
\hline Co-efficient of concentration & 0.364 & 0.352 & 0.364 & 0.352 \\
\hline
\end{tabular}


Table A27 Average real private income, cash transfers and disposable income of income units in $65+$ age group

\begin{tabular}{|c|c|c|c|c|c|c|}
\hline & $\begin{array}{l}\text { Private } \\
\text { income }\end{array}$ & $\begin{array}{l}\text { Direct } \\
\text { benefits } \\
\text { received }\end{array}$ & $\begin{array}{l}\text { Direct } \\
\text { taxes } \\
\text { paid }\end{array}$ & $\begin{array}{l}\text { Net cash } \\
\text { transfers }\end{array}$ & $\begin{array}{l}\text { Disposable } \\
\text { income }\end{array}$ & $\begin{array}{l}\text { Disposable } \\
\text { income } \\
\text { as \% of } \\
\text { private } \\
\text { income }\end{array}$ \\
\hline & \multicolumn{5}{|c|}{ Average annual value (\$A) } & $\%$ \\
\hline \multicolumn{7}{|c|}{ Married couples $65+$} \\
\hline Australia & 6,182 & $+6,389$ & $-1,204$ & $+5,185$ & 11,367 & 183.9 \\
\hline Sweden & 3,506 & $+12,912$ & $-4,751$ & $+8,161$ & 11,667 & 332.8 \\
\hline $\begin{array}{l}\text { Australia as \% } \\
\text { of Sweden }\end{array}$ & 176.3 & & & & 97.4 & \\
\hline \multicolumn{7}{|c|}{ Single persons $65+$} \\
\hline Australia & 2,377 & $+4,339$ & -558 & $+3,781$ & 6,158 & 259.1 \\
\hline Sweden & 1,201 & $+6,896$ & $-1,925$ & $+4,971$ & 6,172 & 513.9 \\
\hline $\begin{array}{l}\text { Australia as \% } \\
\text { of Sweden }\end{array}$ & 197.9 & & & & 99.8 & \\
\hline \multicolumn{7}{|c|}{ All income units in $65+$ age group } \\
\hline Australia & 4,061 & $+5,256$ & -844 & $+4,402$ & 8,463 & 208.4 \\
\hline Sweden & 2,056 & $+9,126$ & $-2,973$ & $+6,153$ & 8,209 & 399.3 \\
\hline $\begin{array}{l}\text { Australia as \% } \\
\text { of Sweden }\end{array}$ & 162.1 & & & & 103.1 & \\
\hline
\end{tabular}

Table A28 Income units in 65 years + age group: average real private and disposable income by income quantiles

\begin{tabular}{|c|c|c|c|c|}
\hline \multicolumn{5}{|c|}{ Average annual income } \\
\hline \multirow{2}{*}{ Income quantile } & \multicolumn{2}{|c|}{$\$ A^{\prime} 000$} & \multicolumn{2}{|c|}{$\%$ of total } \\
\hline & Australia & Sweden & Australia & Sweden \\
\hline \multicolumn{5}{|c|}{ Private income, units ranked by private income } \\
\hline Lowest $10 \%$ & 0.0 & -0.1 & 0.0 & -0.5 \\
\hline Second decile & 0.0 & 0.0 & 0.0 & 0.1 \\
\hline Third decile & 0.0 & 0.2 & 0.1 & 0.8 \\
\hline Fourth decile & 0.2 & 0.3 & 0.4 & 1.6 \\
\hline Fifth decile & 0.4 & 0.5 & 1.1 & 2.6 \\
\hline Sixth decile & 0.9 & 0.9 & 2.1 & 4.2 \\
\hline Seventh decile & 1.6 & 1.3 & 3.8 & 6.3 \\
\hline Eighth decile & 3.0 & 1.9 & 7.4 & 9.5 \\
\hline Ninth decile & 8.4 & 3.5 & 20.7 & 17.0 \\
\hline Highest $10 \%$ & 26.1 & 12.0 & 64.4 & 58.4 \\
\hline Highest $5 \%$ & 35.4 & 17.8 & 43.7 & 43.2 \\
\hline All income units & 4.1 & 2.1 & 100.0 & 100.0 \\
\hline Co-efficient of concentration & 0.772 & 0.708 & 0.772 & 0.708 \\
\hline \multicolumn{5}{|c|}{ Disposable income, units ranked by disposable income } \\
\hline Lowest $5 \%$ & 3.3 & 3.8 & 2.0 & 2.3 \\
\hline Lowest $10 \%$ & 3.9 & 4.1 & 4.6 & 5.0 \\
\hline Second decile & 4.7 & 4.9 & 5.6 & 5.9 \\
\hline Third decile & 5.0 & 5.5 & 5.9 & 6.6 \\
\hline
\end{tabular}




\begin{tabular}{|l|c|c|c|c|}
\hline Fourth decile & 5.4 & 5.9 & 6.4 & 7.2 \\
\hline Fifth decile & 6.6 & 6.6 & 7.8 & 8.0 \\
\hline Sixth decile & 7.9 & 7.6 & 9.3 & 9.2 \\
\hline Seventh decile & 8.6 & 8.8 & 10.1 & 10.7 \\
\hline Eighth decile & 9.7 & 10.0 & 11.5 & 12.1 \\
\hline Ninth decile & 11.7 & 11.7 & 13.8 & 14.2 \\
\hline Highest 10\% & 21.0 & 17.2 & 25.0 & 21.1 \\
\hline Highest 5\% & 26.1 & 20.6 & 15.7 & 12.6 \\
\hline Highest 2.5\% & 31.8 & 25.4 & 9.5 & 7.7 \\
\hline All income units & 8.5 & 8.2 & 100.0 & 100.0 \\
\hline Co-efficient of concentration & 0.281 & 0.242 & 0.281 & 0.242 \\
\hline
\end{tabular}

\section{Table A29 Terms used in Australia/Sweden comparisons}

\begin{tabular}{|c|c|c|}
\hline & $\begin{array}{l}\text { As used in tables } \\
\text { 'Australia and Sweden 1984' }\end{array}$ & $\begin{array}{l}\text { As used in Income Distribution } \\
\text { Survey publications of } \\
\text { Statistics Sweden }\end{array}$ \\
\hline Income concepts & $\begin{array}{l}\text { Private income } \\
\text { Direct benefits received } \\
\text { Direct taxes paid } \\
\text { Disposable income }\end{array}$ & $\begin{array}{l}\text { Factor income } \\
\text { Positive transfers } \\
\text { Negative transfers } \\
\text { Disposable income }\end{array}$ \\
\hline \multirow[t]{2}{*}{ Income unit (IU) } & $\begin{array}{l}\text { Income unit } \\
20-64 \text { age group:Married couple } \\
\text { without children } \\
\text { Married couple with children } \\
\text { Single parents } \\
\text { Single persons without children } \\
65+\text { age group:Married couple } \\
\text { Single persons } \\
18-19 \text { age group }\end{array}$ & $\begin{array}{l}\text { Family unit } \\
20-64 \text { age group:Cohabitants } \\
\text { without children } \\
\text { Cohabitants with children } \\
\text { Single person with children } \\
\text { Single persons without children } \\
65+\text { age group:Cohabitants } \\
\text { Single persons } \\
18-19 \text { age group }\end{array}$ \\
\hline & \multicolumn{2}{|c|}{$\begin{array}{l}\text { Note: 'Married couple' includes both legal and defacto relationships as } \\
\text { identified by survey respondents. } \\
\text { 'Children' are persons under } 18 \text { years. }\end{array}$} \\
\hline $\begin{array}{l}\text { Consumption } \\
\text { unit (CU) }\end{array}$ & $\begin{array}{l}\text { Married couple }=1 \mathrm{CU} \\
\text { Single person }=0.6 \mathrm{CU} \\
\text { Children }=0.25 \mathrm{CU}\end{array}$ & \\
\hline $\begin{array}{l}\text { Employment } \\
\text { status }\end{array}$ & $\begin{array}{l}\text { Income unit with: } \\
0 \text { employed } \\
1 \text { employed } \\
2 \text { employed } \\
1+\text { employed } \\
\end{array}$ & $\begin{array}{l}\text { Family unit: } \\
\text { Economically inactive } \\
\text { With } 1 \text { economically active } \\
\text { With } 2 \text { economically active } \\
\text { Economically active }\end{array}$ \\
\hline $\begin{array}{l}\text { Incomes, } \\
\text { benefits and } \\
\text { taxes }\end{array}$ & \multicolumn{2}{|c|}{$\begin{array}{l}\text { Average weekly values for Australian income units from the ABS } \\
\text { Household Expenditure Survey } 1984 \text { are converted to annual levels by } \\
\text { multiplying by } 52 \text {. } \\
\text { Average annual values for Swedish 'family units', as given in Income } \\
\text { Distribution Survey publications for } 1984 \text {, are converted to \$A } \\
\text { assuming a PPP ratio of SKr } 7.2=\$ \mathrm{~A} 1 \text {. } \\
\text { Note: Employment of children (persons under } 18 \text { years) is disregarded } \\
\text { in assessing the employment status of income units. }\end{array}$} \\
\hline
\end{tabular}




\section{Attachment B}

\section{Australia and United States 1984: List of tables}

B: Distribution of total household income among households: period income from combined data from the Annual Housing Survey, the Income Survey Development Program and the Internal Revenue Service, as well as the March 1985 Current Population Survey (CPS) conducted by the Bureau of the Census, and current income from Australian 1984 Household Expenditure Survey.

\section{All households}

By household type

B1 Numbers of households, persons and consumption units

B2 Average real income before and after tax per household and consumption unit

By age of household head

B3 Numbers of households and persons

B4 All households: Average real income before and after tax per household and per person

\section{By nature of housing occupancy}

B5 Numbers of households and persons, and average real income fefore and after $\operatorname{tax}$

By household gross income deciles

B6 Average real income before and after tax per household and per person

B7 Direct taxes as \% of gross incomes, by type of tax

\section{Direct taxes}

B8 Direct taxes as \% of gross income, by household type, age of household head, nature of housing occupancy and household gross income decile

\section{Definitions of terms}

B9 Terms used in Australia/United States comparisons 
Table B1 All households: numbers of households, persons and consumption units, by household type

\begin{tabular}{|c|c|c|}
\hline & Australia & $\begin{array}{l}\text { United } \\
\text { States }\end{array}$ \\
\hline Number of households ('000) & 5,039 & 86,789 \\
\hline Of which - & $\%$ & $\%$ \\
\hline Married couples with no children under 18 & 30.6 & 29.2 \\
\hline Married couples with children under 18 & 36.5 & 28.8 \\
\hline Female householder, no husband present, with children under 18 & 4.5 & 7.9 \\
\hline Single person households & 19.1 & 23.7 \\
\hline \multirow[t]{2}{*}{ All other } & 9.3 & 10.4 \\
\hline & 100.0 & 100.0 \\
\hline Number of persons in households ('000) & 14,291 & 233,516 \\
\hline Of which in households consisting of - & $\%$ & $\%$ \\
\hline Married couples with no children under 18 & 24.8 & 25.8 \\
\hline Married couples with children under 18 & 54.9 & 45.2 \\
\hline Female householder, no husband present, with children under 18 & 4.9 & 10.0 \\
\hline Single person households & 6.7 & 8.8 \\
\hline \multirow[t]{2}{*}{ All other } & 8.7 & 10.2 \\
\hline & 100.0 & 100.0 \\
\hline Number of consumption units in households ( $(000)$ & 6,378 & 108,452 \\
\hline Of which in households consisting of - & $\%$ & $\%$ \\
\hline Married couples with no children under 18 & 28.4 & 28.7 \\
\hline Married couples with children under 18 & 47.2 & 38.5 \\
\hline Female householder, no husband present, with children under 18 & 4.4 & 8.8 \\
\hline Single person households & 9.0 & 11.4 \\
\hline \multirow[t]{2}{*}{ All other } & 10.9 & 12.6 \\
\hline & 100.0 & 100.0 \\
\hline
\end{tabular}

Table B2 All households: average real income before and after tax per household and consumption unit, by household type

\begin{tabular}{|c|c|c|}
\hline & Australia & United States \\
\hline Average real income per household before tax: & $\$ A 23,587$ & $\$ A 32,683$ \\
\hline \multicolumn{3}{|c|}{ Ratio: Average for all households $=100-$} \\
\hline Married couples with no children under 18 & 107.0 & 123.7 \\
\hline Married couples with children under 18 & 121.0 & 125.6 \\
\hline $\begin{array}{l}\text { Female householder, no husband present, with } \\
\text { children under } 18\end{array}$ & 60.2 & 51.6 \\
\hline Single person households & 49.5 & 55.8 \\
\hline All other & 117.5 & 100.0 \\
\hline
\end{tabular}




\begin{tabular}{|c|c|c|}
\hline & Australia & United States \\
\hline Average real income per household after tax: & $\$ A 18,617$ & $\$ A 5,664$ \\
\hline \multicolumn{3}{|c|}{ Ratio: Average for all households $=100-$} \\
\hline Married couples with no children under 18 & 107.2 & 122.7 \\
\hline Married couples with children under 18 & 119.2 & 124.5 \\
\hline $\begin{array}{l}\text { Female householder, no husband present, with } \\
\text { children under } 18\end{array}$ & 67.1 & 56.8 \\
\hline Single person households & 49.7 & 56.3 \\
\hline All other & 119.9 & 101.0 \\
\hline $\begin{array}{l}\text { Average real income per consumption unit } \\
\text { before tax: }\end{array}$ & $\$ A 18,636$ & $\$ A 26,157$ \\
\hline \multicolumn{3}{|c|}{ Ratio: Average for all households $=100-$} \\
\hline Married couples with no children under 18 & 115.1 & 125.6 \\
\hline Married couples with children under 18 & 93.5 & 94.1 \\
\hline $\begin{array}{l}\text { Female householder, no husband present, with } \\
\text { children under } 18\end{array}$ & 61.4 & 46.2 \\
\hline Single person households & 104.3 & 116.2 \\
\hline All other & 100.6 & 82.6 \\
\hline $\begin{array}{l}\text { Average real income per consumption unit } \\
\text { after tax: }\end{array}$ & $\$ A 14,709$ & $\$ A 20,538$ \\
\hline \multicolumn{3}{|c|}{ Ratio: Average for all households $=100-$} \\
\hline Married couples with no children under 18 & 115.4 & 124.6 \\
\hline Married couples with children under 18 & 92.1 & 93.2 \\
\hline $\begin{array}{l}\text { Female householder, no husband present, with } \\
\text { children under } 18\end{array}$ & 68.4 & 50.8 \\
\hline Single persons households & 104.9 & 117.2 \\
\hline All other & 102.7 & 83.5 \\
\hline
\end{tabular}

Table B3 All households: numbers of households and persons, by age of household head

\begin{tabular}{|l|c|c|}
\hline & Australia & United States \\
\hline Number of households ('000) & $\mathbf{5 , 0 3 9}$ & $\mathbf{8 6 , 7 8 9}$ \\
\hline Of which age of household head: & $\%$ & $\%$ \\
Under 25 years & 6.8 & 6.3 \\
25-34 years & 22.4 & 23.1 \\
$\mathbf{3 5 - 4 4}$ years & 20.8 & 20.1 \\
$\mathbf{4 5 - 5 4}$ years & 15.0 & 14.5 \\
$\mathbf{5 5 - 6 4}$ years & 16.0 & 15.1 \\
$\mathbf{6 5}$ years and over & 19.0 & 20.9 \\
\hline & 100.0 & 100.0 \\
\hline Number of persons in households ('000) & $\mathbf{1 4 , 2 9 1}$ & $\mathbf{2 3 3 , 5 1 6}$ \\
\hline Of which in households with head aged: & $\%$ & $\%$ \\
Under 25 years & 5.5 & 24.5 \\
$\mathbf{2 5 - 3 4}$ years & 24.5 & 25.9 \\
$\mathbf{3 5 - 4 4}$ years & 28.5 & 17.0 \\
$\mathbf{4 5 - 5 4}$ years & 17.2 & 13.4 \\
$\mathbf{5 5 - 6 4}$ years & 12.8 & 13.8 \\
$\mathbf{6 5}$ years and over & 11.5 & 100.0 \\
\hline
\end{tabular}


Measuring and Promoting Wellbeing: How Important is Economic Growth?

Table B4 All households: average real income before and after tax per household and per person, by age of household head

\begin{tabular}{|c|c|c|}
\hline & Australia & United States \\
\hline Average real income per household before tax & $\$ A 23,587$ & $\$ A 32,683$ \\
\hline \multicolumn{3}{|c|}{ Ratio: Average for all households $=100-$} \\
\hline $\begin{array}{l}\text { Under } 25 \text { years } \\
25-34 \text { years } \\
35-44 \text { years } \\
45-54 \text { years } \\
55-64 \text { years } \\
65 \text { years and over }\end{array}$ & $\begin{array}{c}94.0 \\
108.0 \\
119.3 \\
134.0 \\
92.8 \\
50.6 \\
\end{array}$ & $\begin{array}{c}60.6 \\
95.3 \\
121.6 \\
131.1 \\
111.1 \\
66.6\end{array}$ \\
\hline Average real income per household after tax & $\$ A 18,617$ & $\$ A 25,664$ \\
\hline \multicolumn{3}{|c|}{ Ratio: Average for all households $=100-$} \\
\hline $\begin{array}{l}\text { Under } 25 \text { years } \\
25-34 \text { years } \\
35-44 \text { years } \\
45-54 \text { years } \\
45-64 \text { years } \\
65 \text { years and over }\end{array}$ & $\begin{array}{c}95.9 \\
106.3 \\
116.4 \\
130.7 \\
94.8 \\
56.0 \\
\end{array}$ & $\begin{array}{c}63.6 \\
95.2 \\
118.7 \\
127.2 \\
108.7 \\
73.0 \\
\end{array}$ \\
\hline Average real income per person before tax & $\$ A 8,305$ & $\$ A 12,150$ \\
\hline \multicolumn{3}{|c|}{ Ratio: Average income per person before tax $=100-$} \\
\hline $\begin{array}{l}\text { Under } 25 \text { years } \\
25-34 \text { years } \\
35-44 \text { years } \\
45-54 \text { years } \\
55-64 \text { years } \\
65 \text { years and over }\end{array}$ & $\begin{array}{c}117.4 \\
99.00 \\
87.1 \\
117.0 \\
115.1 \\
83.4 \\
\end{array}$ & $\begin{array}{c}70.1 \\
89.6 \\
94.5 \\
112.0 \\
125.5 \\
101.1 \\
\end{array}$ \\
\hline Average real income per person after tax & $\$ A 6,565$ & $\$ A 9,541$ \\
\hline \multicolumn{3}{|c|}{ Ratio: Average income per person after tax $=100-$} \\
\hline $\begin{array}{l}\text { Under } 25 \text { years } \\
25-34 \text { years } \\
35-44 \text { years } \\
45-54 \text { years } \\
55-64 \text { years } \\
65 \text { years and over }\end{array}$ & $\begin{array}{c}119.8 \\
97.4 \\
81.1 \\
114.2 \\
117.6 \\
92.4 \\
\end{array}$ & $\begin{array}{c}73.6 \\
89.5 \\
92.2 \\
108.7 \\
122.7 \\
110.9 \\
\end{array}$ \\
\hline
\end{tabular}


Table B5 All households: numbers of households and persons, and average real income before and after tax per household and per person, by nature of housing occupancy

\begin{tabular}{|c|c|c|}
\hline & Australia & United States \\
\hline Number of households ('000) & 5,039 & 86,789 \\
\hline Of which - & $\%$ & $\%$ \\
\hline \multirow[t]{2}{*}{$\begin{array}{l}\text { Dwelling owned outright or being bought } \\
\text { Dwelling rented or occupied rent free }\end{array}$} & $\begin{array}{l}71.5 \\
28.5\end{array}$ & $\begin{array}{l}64.3 \\
35.7\end{array}$ \\
\hline & 100.0 & 100.0 \\
\hline Number of persons in households ('000) & 14,291 & 233,516 \\
\hline \multicolumn{3}{|l|}{ Of which - } \\
\hline \multirow[t]{3}{*}{$\begin{array}{l}\text { Dwelling owned outright or being bought } \\
\text { Dwelling rented or occupied rent free }\end{array}$} & $\begin{array}{l}73.7 \\
26.3\end{array}$ & $\begin{array}{l}68.1 \\
31.9\end{array}$ \\
\hline & 100.0 & 100.0 \\
\hline & $\$ \mathrm{~A}$ & $\$ \mathrm{~A}$ \\
\hline Average real income per household before tax & $\$ A 23,587$ & $\$ A 32,683$ \\
\hline \multicolumn{3}{|c|}{ Ratio: Average for all households $=100-$} \\
\hline $\begin{array}{l}\text { Households in owned dwellings } \\
\text { Households in rented dwellings }\end{array}$ & $\begin{array}{c}105.5 \\
86.1\end{array}$ & $\begin{array}{c}116.7 \\
69.8 \\
\end{array}$ \\
\hline Average real income per household after tax & $\$ A 86,617$ & $\$ A 25,664$ \\
\hline \multicolumn{3}{|c|}{ Ratio: Average for all households $=100-$} \\
\hline $\begin{array}{l}\text { Households in owned dwellings } \\
\text { Households in rented dwellings }\end{array}$ & $\begin{array}{c}104.8 \\
88.1\end{array}$ & $\begin{array}{c}115.3 \\
71.9\end{array}$ \\
\hline Average real income per person before tax & $\$ A 8,305$ & $\$ A 12,150$ \\
\hline \multicolumn{3}{|c|}{ Ratio: Average before tax income per person $=100-$} \\
\hline $\begin{array}{l}\text { Persons in owned dwellings } \\
\text { Persons in rented dwellings }\end{array}$ & $\begin{array}{l}102.6 \\
93.4\end{array}$ & $\begin{array}{l}110.3 \\
78.0\end{array}$ \\
\hline Average real income per person after tax & $\$ A 6,565$ & $\$ A 9,541$ \\
\hline \multicolumn{3}{|c|}{ Ratio: Average after tax income per person $=100-$} \\
\hline $\begin{array}{l}\text { Persons in owned dwellings } \\
\text { Persons in rented dwellings }\end{array}$ & $\begin{array}{l}101.7 \\
95.3\end{array}$ & $\begin{array}{c}109.0 \\
80.8 \\
\end{array}$ \\
\hline
\end{tabular}


Measuring and Promoting Wellbeing: How Important is Economic Growth?

Table B6 All households: average real income before and after tax per household and per person, by household gross income deciles

\begin{tabular}{|c|c|c|}
\hline & Australia & United States \\
\hline Average real income per household before tax & $\$ A 23,587$ & $\$ A 32,683$ \\
\hline \multicolumn{3}{|c|}{ Ratio: Average for all households $=100-$} \\
\hline $\begin{array}{l}\text { Lowest } 10 \% \\
\text { Second decile } \\
\text { Third decile } \\
\text { Fourth decile } \\
\text { Fifth decile } \\
\text { Sixth decile } \\
\text { Seventh decile } \\
\text { Eighth decile } \\
\text { Ninth decile } \\
\text { Highest } 10 \% \\
\text { Co-efficient of concentration }\end{array}$ & $\begin{array}{l}19 \\
32 \\
44 \\
61 \\
77 \\
94 \\
114 \\
137 \\
167 \\
255 \\
0.370 \\
\end{array}$ & $\begin{array}{c}12 \\
27 \\
42 \\
57 \\
73 \\
91 \\
110 \\
136 \\
173 \\
279 \\
0.407 \\
\end{array}$ \\
\hline Average real income per household after tax & $\$ A 18,617$ & $\$ A 25,664$ \\
\hline \multicolumn{3}{|c|}{ Ratio: Average for all households $=100-$} \\
\hline $\begin{array}{l}\text { Lowest } 10 \% \\
\text { Second decile } \\
\text { Third decile } \\
\text { Fourth decile } \\
\text { Fifth decile } \\
\text { Sixth decile } \\
\text { Seventh decile } \\
\text { Eighth decile } \\
\text { Ninth decile } \\
\text { Highest } 10 \% \\
\text { Co-efficient of concentration } \\
\end{array}$ & $\begin{array}{c}23 \\
40 \\
53 \\
68 \\
82 \\
97 \\
114 \\
134 \\
161 \\
228 \\
0.325 \\
\end{array}$ & $\begin{array}{c}14 \\
33 \\
48 \\
63 \\
78 \\
94 \\
113 \\
136 \\
168 \\
253 \\
0.370 \\
\end{array}$ \\
\hline Average real income per person before tax & $\$ A 8,305$ & $\$ A 12,150$ \\
\hline \multicolumn{3}{|c|}{ Ratio: Average income before tax per person $=100-$} \\
\hline $\begin{array}{l}\text { Lowest } 10 \% \\
\text { Second decile } \\
\text { Third decile } \\
\text { Fourth decile } \\
\text { Fifth decile } \\
\text { Sixth decile } \\
\text { Seventh decile } \\
\text { Eighth decile } \\
\text { Ninth decile } \\
\text { Highest } 10 \% \\
\end{array}$ & $\begin{array}{c}41 \\
45 \\
48 \\
64 \\
72 \\
86 \\
102 \\
212 \\
142 \\
190 \\
\end{array}$ & \begin{tabular}{|c|}
17 \\
36 \\
49 \\
64 \\
75 \\
86 \\
100 \\
117 \\
143 \\
223 \\
\end{tabular} \\
\hline Average real income per person after tax & $\$ A 6,565$ & \$A9,541 \\
\hline \multicolumn{3}{|c|}{ Ratio: Average income after tax per person $=100-$} \\
\hline $\begin{array}{l}\text { Lowest } 10 \% \\
\text { Second decile } \\
\text { Third decile } \\
\text { Fourth decile } \\
\text { Fifth decile } \\
\text { Sixth decile } \\
\text { Seventh decile } \\
\text { Eighth decile } \\
\text { Ninth decile } \\
\text { Highest } 10 \% \\
\end{array}$ & $\begin{array}{c}50 \\
55 \\
57 \\
70 \\
77 \\
89 \\
102 \\
119 \\
137 \\
170 \\
\end{array}$ & $\begin{array}{c}20 \\
43 \\
56 \\
70 \\
80 \\
90 \\
102 \\
117 \\
139 \\
202 \\
\end{array}$ \\
\hline
\end{tabular}


Table B7 All households: direct taxes as percentage of gross incomes, by type of tax and gross income decile

\begin{tabular}{|c|c|c|c|c|c|}
\hline & $\begin{array}{l}\text { Federal } \\
\text { income tax }\end{array}$ & $\begin{array}{l}\text { State } \\
\text { income } \\
\text { taxes }\end{array}$ & $\begin{array}{l}\text { Payroll } \\
\text { taxes on } \\
\text { households }\end{array}$ & $\begin{array}{l}\text { Property } \\
\text { taxes }\end{array}$ & $\begin{array}{l}\text { All } \\
\text { direct } \\
\text { taxes }\end{array}$ \\
\hline & $\%$ & $\%$ & $\%$ & $\%$ & $\%$ \\
\hline \multicolumn{6}{|c|}{ Lowest $10 \%$} \\
\hline Australia & 0.4 & - & - & 3.0 & 3.4 \\
\hline United States & 0.3 & 0.2 & 1.8 & 4.8 & 7.1 \\
\hline \multicolumn{6}{|c|}{ Second decile } \\
\hline Australia & 1.7 & - & - & 1.9 & 3.6 \\
\hline United States & 1.2 & 0.4 & 2.5 & 3.0 & 7.1 \\
\hline \multicolumn{6}{|c|}{ Third decile } \\
\hline Australia & 3.8 & - & - & 1.5 & 5.3 \\
\hline United States & 3.5 & 0.9 & 3.7 & 2.3 & 10.4 \\
\hline \multicolumn{6}{|c|}{ Fourth decile } \\
\hline Australia & 11.6 & - & - & 1.1 & 12.7 \\
\hline United States & 5.7 & 1.3 & 4.3 & 2.1 & 13.4 \\
\hline \multicolumn{6}{|c|}{ Fifth decile } \\
\hline Australia & 15.0 & - & - & 1.1 & 16.1 \\
\hline United States & 7.4 & 1.7 & 4.8 & 1.8 & 15.7 \\
\hline \multicolumn{6}{|c|}{ Sixth decile } \\
\hline Australia & 17.8 & - & - & 0.9 & 18.7 \\
\hline United States & 9.0 & 2.1 & 5.3 & 1.8 & 18.2 \\
\hline \multicolumn{6}{|c|}{ Seventh decile } \\
\hline Australia & 20.2 & - & - & 0.8 & 21.0 \\
\hline United States & 10.0 & 2.6 & 5.4 & 1.7 & 19.7 \\
\hline \multicolumn{6}{|c|}{ Eighth decile } \\
\hline Australia & 21.6 & - & - & 0.8 & 22.4 \\
\hline United States & 11.5 & 2.8 & 5.7 & 1.7 & 21.7 \\
\hline \multicolumn{6}{|c|}{ Ninth decile } \\
\hline Australia & 23.2 & - & - & 0.7 & 23.9 \\
\hline United States & 13.9 & 3.2 & 5.1 & 1.5 & 23.7 \\
\hline \multicolumn{6}{|c|}{ Highest $10 \%$} \\
\hline Australia & 28.6 & - & - & 0.5 & 29.1 \\
\hline United States & 18.7 & 4.0 & 4.6 & 1.4 & 28.7 \\
\hline \multicolumn{6}{|c|}{ All households } \\
\hline Australia & 20.2 & - & - & 0.9 & 21.1 \\
\hline United States & 12.1 & 2.8 & 4.9 & 1.7 & 21.5 \\
\hline
\end{tabular}


Table B8 All households: direct taxes as \% of gross income, by household type, age of household head, nature of housing occupancy and household gross income decile

\begin{tabular}{|c|c|c|}
\hline & Australia \% & United States \% \\
\hline \multicolumn{3}{|c|}{ Household type: } \\
\hline Married couple with no children under 18 & 20.9 & 22.2 \\
\hline Married couple with children under 18 & 22.2 & 22.2 \\
\hline $\begin{array}{l}\text { Female householder, no husband present, with } \\
\text { children under } 18\end{array}$ & 12.0 & 13.7 \\
\hline Single person households & 20.6 & 20.8 \\
\hline All other & 19.4 & 20.7 \\
\hline All households & 21.1 & 21.5 \\
\hline \multicolumn{3}{|c|}{ Age of household head: } \\
\hline Under 25 years & 19.5 & 17.5 \\
\hline $25-34$ years & 22.4 & 21.6 \\
\hline $35-44$ years & 22.9 & 23.4 \\
\hline $45-54$ years & 23.0 & 23.8 \\
\hline $55-64$ years & 19.4 & 23.2 \\
\hline 65 years and over & 12.6 & 13.9 \\
\hline All households & 21.1 & 21.5 \\
\hline \multicolumn{3}{|c|}{ Nature of housing occupancy: } \\
\hline Owned outright or being bought & 21.7 & 22.4 \\
\hline Rented or occupied rent free & 19.3 & 19.1 \\
\hline All households & 21.1 & 21.5 \\
\hline \multicolumn{3}{|c|}{ Gross income decile: } \\
\hline Lowest $10 \%$ & 3.4 & 7.1 \\
\hline Second decile & 3.6 & 7.1 \\
\hline Third decile & 5.3 & 10.4 \\
\hline Fourth decile & 12.7 & 13.4 \\
\hline Fifth decile & 16.1 & 15.7 \\
\hline Sixth decile & 18.7 & 18.2 \\
\hline Seventh decile & 21.0 & 19.7 \\
\hline Eighth decile & 22.4 & 21.7 \\
\hline Ninth decile & 23.9 & 23.7 \\
\hline Highest $10 \%$ & 29.1 & 28.7 \\
\hline All households & 21.1 & 21.5 \\
\hline
\end{tabular}




\section{Table B9 Terms used in Australia/United States comparisons}

\begin{tabular}{|c|c|c|}
\hline & $\begin{array}{l}\text { As used in tables 'Australia } \\
\text { and United States } 1984 \text { ' }\end{array}$ & $\begin{array}{l}\text { As used in ABS Household } \\
\text { Expenditure Survey 1984: The } \\
\text { Effects of Government Benefits } \\
\text { and Taxes on Household Income }\end{array}$ \\
\hline Income & $\begin{array}{l}\text { Before-tax income } \\
\text { Direct taxes } \\
\text { After tax income }\end{array}$ & $\begin{array}{l}\text { Gross income } \\
\text { Direct taxes plus expenditure on } \\
\text { general rates from HES } \\
\text { Disposable income less } \\
\text { expenditure on general rates } \\
\text { from HES }\end{array}$ \\
\hline \multirow[t]{2}{*}{ Household } & $\begin{array}{l}\text { Households: } \\
\text { Married couple with no } \\
\text { children under } 18 \\
\text { Married couple with } \\
\text { children under } 18 \\
\text { Female householder, no } \\
\text { husband present, with } \\
\text { children under } 18 \\
\text { Single person households } \\
\text { All other }\end{array}$ & $\begin{array}{l}\text { Households: } \\
\text { Married couple only } \\
\text { Married couple with children } \\
\text { under } 18 \\
\text { Single parent: female, with } \\
\text { children under } 18 \\
\text { Single person households } \\
\text { All other (than households } \\
\text { Included above) }\end{array}$ \\
\hline & \multicolumn{2}{|c|}{$\begin{array}{l}\text { Note: United States Census Bureau estimates, which are for } \\
\text { married couples and female householders with no related } \\
\text { children under 18, have not been adjusted. } \\
\text { ABS estimates for married couples or single parents with } \\
\text { dependent children (see description in The Effects of } \\
\text { Government Benefits and Taxes on Household Income: 79) } \\
\text { have been adjusted to include all persons under } 18 \text { years of } \\
\text { age in households. }\end{array}$} \\
\hline Consumption unit (CU) & \multicolumn{2}{|l|}{$\begin{array}{l}\text { Married couple }=1 \mathrm{CU} \\
\text { Single person }=0.6 \mathrm{CU} \\
\text { Children }=0.25 \mathrm{CU}\end{array}$} \\
\hline $\begin{array}{l}\text { Incomes, benefits and } \\
\text { taxes }\end{array}$ & \multicolumn{2}{|c|}{$\begin{array}{l}\text { Average weekly values for Australian income units from the } \\
\text { ABS Household Expenditure Survey } 1984 \text { are converted to } \\
\text { annual levels by multiplying by } 52 \text {. } \\
\text { Average annual values for US households, as given in US } \\
\text { bureau of the Census After-Tax Money Income Estimates of } \\
\text { Households: } 1984 \text {, are converted to \$A assuming a PPP ratio } \\
\text { of \$US0.84 = \$A1. }\end{array}$} \\
\hline
\end{tabular}

